\title{
Stellar Streams Discovered in the Dark Energy Survey
}

N. Shipp ${ }^{1,2,49}$ (1) A. Drlica-Wagner ${ }^{3}$ (1), E. Balbinot ${ }^{4}$, P. Ferguson ${ }^{5}$, D. Erkal ${ }^{4,6}$, T. S. Li ${ }^{3}$, K. Bechtol $^{7}$, V. Belokurov ${ }^{6}$, B. Buncher $^{3}$,

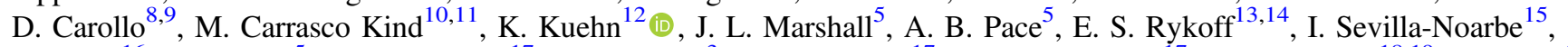
E. Sheldon ${ }^{16}$, L. Strigari ${ }^{5}$ (1) A. K. Vivas ${ }^{17}$ (1) B. Yanny ${ }^{3}$ (1) A. Zenteno ${ }^{17}$, T. M. C. Abbott ${ }^{17}$, F. B. Abdalla ${ }^{18,19}$, S. Allam ${ }^{3}$, S. Avila ${ }^{20,21}$, E. Bertin ${ }^{22,23}$, D. Brooks ${ }^{18}$ (1), D. L. Burke ${ }^{13,14}$, J. Carretero ${ }^{24}$, F. J. Castander ${ }^{25,26}$ (i) R. Cawthon ${ }^{1}$, M. Crocce ${ }^{25,26}$, C. E. Cunha ${ }^{13}$, C. B. D'Andrea ${ }^{27}$, L. N. da Costa ${ }^{28,29}$, C. Davis ${ }^{13}$, J. De Vicente ${ }^{15}$, S. Desai ${ }^{30}$, H. T. Diehl ${ }^{3}$, P. Doel ${ }^{18}$, A. E. Evrard ${ }^{31,32}$, B. Flaugher ${ }^{3}$, P. Fosalba ${ }^{25,26}$, J. Frieman ${ }^{1,3}$, J. García-Bellido ${ }^{21}$ (i), E. Gaztanaga ${ }^{25,26}$, D. W. Gerdes ${ }^{31,32}$, D. Gruen ${ }^{13,14}$ (1) R. A. Gruendl ${ }^{10,11}$ (1) J. Gschwend ${ }^{28,29}$, G. Gutierrez ${ }^{3}$, W. Hartley ${ }^{18,33}$, K. Honscheid ${ }^{34,35}$, B. Hoyle ${ }^{36,37}$, D. J. James ${ }^{38}$, M. D. Johnson ${ }^{11}$, E. Krause ${ }^{39,40}$, N. Kuropatkin ${ }^{3}$, O. Lahav ${ }^{18}$, H. Lin ${ }^{3}$, M. A. G. Maia ${ }^{28,29}$, M. March ${ }^{27}$, P. Martini ${ }^{34,41}$ (1) , F. Menanteau ${ }^{10,11}$, C. J. Miller ${ }^{31,32}$, R. Miquel $^{24,42}$ (1) , R. C. Nichol $^{20}$, A. A. Plazas ${ }^{40}$, A. K. Romer ${ }^{43}$ (1), M. Sako ${ }^{27}$, E. Sanchez ${ }^{15}$, B. Santiago ${ }^{28,44}$, V. Scarpine ${ }^{3}$, R. Schindler ${ }^{14}$, M. Schubnell ${ }^{32}$, M. Smith ${ }^{45}$, R. C. Smith ${ }^{17}$, F. Sobreira ${ }^{28,46}$, E. Suchyta ${ }^{47}$, M. E. C. Swanson ${ }^{11}$, G. $\operatorname{Tarle}^{32}$ (1) D. Thomas $^{20}$, D. L. Tucker $^{3}$, A. R. Walker ${ }^{17}$ (1) and R. H. Wechsler ${ }^{13,14,48}$ (i)

(DES Collaboration)

${ }^{1}$ Kavli Institute for Cosmological Physics, University of Chicago, Chicago, IL 60637, USA; norashipp@uchicago.edu

${ }^{2}$ Department of Astronomy and Astrophysics, University of Chicago, Chicago, IL 60637, USA

${ }^{3}$ Fermi National Accelerator Laboratory, P.O. Box 500, Batavia, IL 60510, USA; kadrlica@fnal.gov

${ }^{4}$ Department of Physics, University of Surrey, Guildford GU2 7XH, UK

${ }^{5}$ George P. and Cynthia Woods Mitchell Institute for Fundamental Physics and Astronomy, and Department of Physics and Astronomy, Texas A\&M University, College Station, TX 77843, USA

${ }^{6}$ Institute of Astronomy, University of Cambridge, Madingley Road, Cambridge CB3 OHA, UK

${ }^{7}$ LSST, 933 North Cherry Avenue, Tucson, AZ 85721, USA

${ }^{8}$ ARC Centre of Excellence for All-sky Astrophysics (CAASTRO), Australia

${ }^{9}$ INAF-Osservatorio Astrofisico di Torino, Pino Torinese, Italy

${ }^{10}$ Department of Astronomy, University of Illinois at Urbana-Champaign, 1002 W. Green Street, Urbana, IL 61801, USA

${ }^{11}$ National Center for Supercomputing Applications, 1205 West Clark Street, Urbana, IL 61801, USA

${ }^{12}$ Australian Astronomical Observatory, North Ryde, NSW 2113, Australia

${ }^{13}$ Kavli Institute for Particle Astrophysics \& Cosmology, P.O. Box 2450, Stanford University, Stanford, CA 94305, USA

${ }^{14}$ SLAC National Accelerator Laboratory, Menlo Park, CA 94025, USA

${ }^{15}$ Centro de Investigaciones Energéticas, Medioambientales y Tecnológicas (CIEMAT), Madrid, Spain ${ }^{16}$ Brookhaven National Laboratory, Bldg. 510, Upton, NY 11973, USA

${ }^{17}$ Cerro Tololo Inter-American Observatory, National Optical Astronomy Observatory, Casilla 603, La Serena, Chile

18 Department of Physics \& Astronomy, University College London, Gower Street, London, WC1E 6BT, UK

${ }^{19}$ Department of Physics and Electronics, Rhodes University, P.O. Box 94, Grahamstown, 6140, South Africa

${ }^{20}$ Institute of Cosmology \& Gravitation, University of Portsmouth, Portsmouth, PO1 3FX, UK

${ }^{21}$ Instituto de Fisica Teorica UAM/CSIC, Universidad Autonoma de Madrid, E-28049 Madrid, Spain

${ }^{22}$ CNRS, UMR 7095, Institut d'Astrophysique de Paris, F-75014, Paris, France

${ }^{23}$ Sorbonne Universités, UPMC Univ Paris 06, UMR 7095, Institut d'Astrophysique de Paris, F-75014, Paris, France

${ }^{24}$ Institut de Física d'Altes Energies (IFAE), The Barcelona Institute of Science and Technology, Campus UAB, E-08193 Bellaterra (Barcelona) Spain ${ }_{25}$ Institut d'Estudis Espacials de Catalunya (IEEC), E-08193 Barcelona, Spain

${ }^{26}$ Institute of Space Sciences (ICE, CSIC), Campus UAB, Carrer de Can Magrans, s/n, E-08193 Barcelona, Spain

${ }^{27}$ Department of Physics and Astronomy, University of Pennsylvania, Philadelphia, PA 19104, USA

${ }^{28}$ Laboratório Interinstitucional de e-Astronomia-LIneA, Rua Gal. José Cristino 77, Rio de Janeiro, RJ-20921-400, Brazil

${ }^{29}$ Observatório Nacional, Rua Gal. José Cristino 77, Rio de Janeiro, RJ-20921-400, Brazil

${ }^{30}$ Department of Physics, IIT Hyderabad, Kandi, Telangana 502285, India

${ }^{31}$ Department of Astronomy, University of Michigan, Ann Arbor, MI 48109, USA

32 Department of Physics, University of Michigan, Ann Arbor, MI 48109, USA

${ }^{33}$ Department of Physics, ETH Zurich, Wolfgang-Pauli-Strasse 16, CH-8093 Zurich, Switzerland

${ }^{34}$ Center for Cosmology and Astro-Particle Physics, The Ohio State University, Columbus, OH 43210, USA

${ }^{35}$ Department of Physics, The Ohio State University, Columbus, OH 43210, USA

${ }^{36}$ Max Planck Institute for Extraterrestrial Physics, Giessenbachstrasse, D-85748 Garching, Germany

${ }^{37}$ Universitäts-Sternwarte, Fakultät für Physik, Ludwig-Maximilians Universität München, Scheinerstr. 1, D-81679 München, Germany

${ }^{38}$ Harvard-Smithsonian Center for Astrophysics, MS-42, 60 Garden Street, Cambridge, MA 02138, USA

${ }^{39}$ Department of Astronomy/Steward Observatory, 933 North Cherry Avenue, Tucson, AZ 85721-0065, USA

${ }^{40}$ Jet Propulsion Laboratory, California Institute of Technology, 4800 Oak Grove Drive, Pasadena, CA 91109, USA

${ }^{41}$ Department of Astronomy, The Ohio State University, Columbus, OH 43210, USA

${ }^{42}$ Institució Catalana de Recerca i Estudis Avançats, E-08010 Barcelona, Spain
Department of Physics and Astronomy, Pevensey Building, University of Sussex, Brighton, BN1 9QH, UK

${ }^{44}$ Instituto de Física, UFRGS, Caixa Postal 15051, Porto Alegre, RS-91501-970, Brazil

${ }^{45}$ School of Physics and Astronomy, University of Southampton, Southampton, SO17 1BJ, UK

${ }^{46}$ Instituto de Física Gleb Wataghin, Universidade Estadual de Campinas, 13083-859, Campinas, SP, Brazil

${ }^{47}$ Computer Science and Mathematics Division, Oak Ridge National Laboratory, Oak Ridge, TN 37831, USA

${ }_{48}$ Department of Physics, Stanford University, 382 Via Pueblo Mall, Stanford, CA 94305, USA

Received 2018 January 9; revised 2018 June 5; accepted 2018 June 12; published 2018 July 30

\footnotetext{
${ }^{49}$ LSSTC Data Science Fellow.
} 


\begin{abstract}
We perform a search for stellar streams around the Milky Way using the first $3 \mathrm{yr}$ of multiband optical imaging data from the Dark Energy Survey (DES). We use DES data covering $\sim 5000 \mathrm{deg}^{2}$ to a depth of $g>23.5$ with a relative photometric calibration uncertainty of $<1 \%$. This data set yields unprecedented sensitivity to the stellar density field in the southern celestial hemisphere, enabling the detection of faint stellar streams to a heliocentric distance of $\sim 50 \mathrm{kpc}$. We search for stellar streams using a matched filter in color-magnitude space derived from a synthetic isochrone of an old, metal-poor stellar population. Our detection technique recovers four previously known thin stellar streams: Phoenix, ATLAS, Tucana III, and a possible extension of Molonglo. In addition, we report the discovery of 11 new stellar streams. In general, the new streams detected by DES are fainter, more distant, and lower surface brightness than streams detected by similar techniques in previous photometric surveys. As a by-product of our stellar stream search, we find evidence for extratidal stellar structure associated with four globular clusters: NGC 288, NGC 1261, NGC 1851, and NGC 1904. The ever-growing sample of stellar streams will provide insight into the formation of the Galactic stellar halo, the Milky Way gravitational potential, and the large- and small-scale distribution of dark matter around the Milky Way.
\end{abstract}

Key words: Galaxy: halo - Galaxy: structure - Local Group

Supporting material: animations

\section{Introduction}

Stellar streams produced by the tidal disruption of globular clusters and dwarf galaxies are a prevalent feature of the Milky Way environs (see Newberg \& Carlin 2016, for a recent review). Observations of stellar streams can provide important constraints on the formation of the Milky Way stellar halo (e.g., Johnston 1998; Bullock \& Johnston 2005; Bell et al. 2008), the shape of the Galactic gravitational field (e.g., Johnston et al. 2005; Koposov et al. 2010; Law \& Majewski 2010; Bonaca et al. 2014; Bovy 2014; Gibbons et al. 2014; PriceWhelan et al. 2014; Sanders 2014; Bowden et al. 2015; Küpper et al. 2015; Erkal et al. 2016b; Bovy et al. 2016), and the abundance of low-mass dark matter substructure (e.g., Ibata et al. 2002; Johnston et al. 2002; Carlberg 2009; Yoon et al. 2011; Carlberg 2012; Ngan \& Carlberg 2014; Erkal \& Belokurov 2015a; Carlberg 2016; Sanderson et al. 2016; Sanders et al. 2016; Bovy et al. 2017; Erkal et al. 2017; Sandford et al. 2017). In addition, stellar streams are a direct snapshot of hierarchical structure formation (Peebles 1965; Press \& Schechter 1974; Blumenthal et al. 1984) and support the standard $\Lambda$ CDM cosmological model (Diemand et al. 2008; Springel et al. 2008).

Wide-area, multiband digital sky surveys have been essential for finding and characterizing resolved stellar populations in the Galactic halo. The Sloan Digital Sky Survey (SDSS; York et al. 2000) revolutionized our understanding of the Milky Way stellar halo, both through improved sensitivity to diffuse components (e.g., Carollo et al. 2007, 2010; de Jong et al. 2010; Deason et al. 2011; An et al. 2013, 2015; Hattori et al. 2013; Kafle et al. 2013; Das \& Binney 2016) and by vastly increasing the number of known satellite galaxies (e.g., Willman et al. 2005a, 2005b; Belokurov et al. 2006b, 2007c; Zucker et al. 2006a, 2006b), stellar clouds (e.g., Newberg et al. 2002; Yanny et al. 2003; Rocha-Pinto et al. 2004), and stellar streams (e.g., Odenkirchen et al. 2001; Newberg et al. 2002; Belokurov et al. 2006a; Grillmair 2006). Early techniques for detecting stellar streams used simple color and magnitude cuts to select blue main-sequence turnoff (MSTO) stars (e.g., Grillmair et al. 1995; Belokurov et al. 2006a). More recently, matched-filter techniques have been used to maximize the contrast between distant, metal-poor stellar populations and foreground field stars to push the detection limit to lower surface brightnesses (e.g., Rockosi et al. 2002). The matched-filter technique has been applied broadly to other digital sky surveys, including Pan-STARRS (Bernard et al. 2014, 2016; Grillmair 2017) and ATLAS (Koposov et al. 2014).

The Dark Energy Survey (DES; DES Collaboration 2005,2016 ) is a deep, wide-area survey with the primary goal of constraining dark energy and the nature of cosmic acceleration (e.g., DES Collaboration 2017). While DES was designed to probe the evolution of the universe out to $z \sim 1.2$, it has already had a major impact on "near-field" cosmology and Galactic archeology. Specifically, DES has nearly doubled the number of known ultrafaint dwarf galaxies (Bechtol et al. 2015; Koposov et al. 2015; Drlica-Wagner et al. 2015; Kim \& Jerjen 2015), increased the number of known faint outer-halo star clusters (Luque et al. 2016, 2017, 2018; Pieres et al. 2016), and identified several diffuse stellar overdensities (Li et al. 2016; Pieres et al. 2017). In addition, early data from DES have been used to detect a cold stellar stream in the constellation of Phoenix (Balbinot et al. 2016) and a tidal stream associated with the ultrafaint satellite Tucana III (DrlicaWagner et al. 2015). Here we extend the search for stellar streams with DES using a deeper, more uniform, and bettercalibrated data set.

We perform a search for stellar streams using the first $3 \mathrm{yr}$ of DES data. We search for stellar streams possessing old, metalpoor stellar populations at heliocentric distances between $6 \mathrm{kpc} \lesssim$ $D_{\odot} \lesssim 63 \mathrm{kpc}(14<m-M<19)$. We recover known streams within the DES footprint, including Sagittarius (Ibata et al. 2001; Newberg et al. 2002), ATLAS (Koposov et al. 2014), Phoenix (Balbinot et al. 2016), and Tucana III (Drlica-Wagner et al. 2015). In addition, we detect a possible faint extension of the Molonglo stream (Grillmair 2017) in the DES data. Our search results in the discovery of 11 new high-significance curvilinear stellar stream candidates. These new stream candidates range in distance from $\sim 13$ to $\sim 50 \mathrm{kpc}$. These streams are low surface brightness, $\mu \gtrsim 32 \mathrm{mag} / \operatorname{arcsec}^{2}$, and push the boundary of detectability using the current generation of wide-area photometric surveys. As a natural by-product of a global search for extended stellar structures, we find evidence for extended extratidal features around the Milky Way globular clusters NGC 288, NGC 1261, NGC 1851, and NGC 1904.

This paper is organized as follows. In Section 2 we discuss the DES data and the construction of the stellar sample used in this work. In Section 3 we present our matched-filter search 
algorithm and maximum-likelihood techniques that we implement for characterizing stellar streams. In Section 4 we discuss the properties of previously known and newly detected stellar streams. We place our results in the larger context of the Milky Way in Section 5 and conclude in Section 6.

\section{Data Set}

DES is a deep, wide-area imaging survey using the Dark Energy Camera (DECam; Flaugher et al. 2015) mounted on the $4 \mathrm{~m}$ Blanco Telescope at Cerro Tololo Inter-American Observatory in Chile. DES surveys $\sim 5000 \mathrm{deg}^{2}$ of the southern Galactic cap in five visible/near-infrared filters, grizY. Here, we use wide-field imaging data from an internal data release of the first $3 \mathrm{yr}$ of DES operations (DES Y3A2). ${ }^{50}$ DES Y3A2 is the first data set to cover the full DES wide-area footprint and has a median coverage of five to six exposures per filter (Diehl et al. 2016). The contiguous, uniform, wide-area imaging of DES allows for the first deep, systematic search for faint features in the Milky Way stellar halo in this region of the southern sky.

The DES Y3A2 images were processed by the DES data management pipeline (Morganson et al. 2018). Photometric calibration was performed via the Forward Global Calibration Method (FGCM; Burke et al. 2018), which utilized ancillary information about atmospheric and environmental conditions at the time of each exposure. The FGCM photometric calibration is found to have a relative photometric uniformity of $\sim 7 \mathrm{mmag}$ (Burke et al. 2018) and an absolute calibration accuracy of $\sim 3$ mmag (DES Collaboration 2018). Individual exposures were remapped to a consistent pixel grid and co-added to increase imaging depth (Morganson et al. 2018). Object detection was performed on a combination of the $r+i+z$ co-added images using the SExtractor toolkit (Bertin \& Arnouts 1996; Bertin et al. 2002) with an object detection threshold corresponding to a signal-to-noise ratio of $\sim 10$ (Morganson et al. 2018).

While the co-added images increase our sensitivity to faint sources, depth variations and point-spread function (PSF) discontinuities in the co-adds can make it difficult to perform precise photometric and morphological measurements (e.g., Drlica-Wagner et al. 2018). To circumvent this issue, we used ngmix $^{51}$ (Sheldon 2014; Jarvis et al. 2016; Drlica-Wagner et al. 2018) to fit the flux and morphology of each source over all individual single-epoch images simultaneously. When fitting each source, we masked nearby neighbors using the uberseg map, which was derived from the SExtractor co-add segmentation maps (Section 5.2 of Jarvis et al. 2016). ${ }^{52}$ Throughout this paper, quoted magnitudes and errors were derived from fitting a PSF model to each source using ngmix (i.e., PSF_MAG and PSF_MAG_ERR).

To select a high-quality stellar sample, we expanded on the star-galaxy classification procedure outlined in Appendix A of Rozo et al. (2016). We used ngmix to fit a composite galaxy

\footnotetext{
${ }^{50}$ DES Y3A2 serves as the basis for the first public DES data release (DES DR1; DES Collaboration 2018). However, the internal Y3A2 data release contains improved multiepoch photometric and morphological measurements, as well as other auxiliary data products.

51 https://github.com/esheldon/ngmix

52 Masking nearby sources yields slightly less accurate photometry than the iterative multiobject fitting (MOF) described by Drlica-Wagner et al. (2018); however, masking is less computationally intensive and has a lower failure rate than MOF.
}

model (bulge plus disk) to each source in all bands simultaneously (Drlica-Wagner et al. 2018). We then used the best-fit size, CM_T, and associated uncertainty, CM_T_ERR, from this galaxy-model fit to distinguish point-like objects from those that are spatially extended. Specifically, we defined an extended classification variable, NGMIX_CLASS, based on the sum of three selection criteria,

$$
\begin{aligned}
\text { NGMIX_CLASS }= & \left(\left(\mathrm{CM}_{-} \mathrm{T}+5 \mathrm{CM}{ }^{\mathrm{T}} \_\mathrm{ERR}\right)>0.1\right) \\
& +\left(\left(\mathrm{CM} \_\mathrm{T}+\mathrm{CM} \_\mathrm{T} \_\mathrm{ERR}\right)>0.05\right) \\
& +\left(\left(\mathrm{CM} \_\mathrm{T}-\mathrm{CM}{ }_{-} \mathrm{T} \_\mathrm{ERR}\right)>0.02\right) .
\end{aligned}
$$

The ngmix composite galaxy-model fit fails for a small number of bright stars. To recover those objects, we defined a second selection based on the weighted-average SExtractor quantity WAVG_SPREAD_MODEL measured in the DES $i$-band (Morganson et al. 2018),

$$
\begin{aligned}
& \text { WAVG_CLASS } \\
&=((\text { WAVG_SPREAD_MODEL_I } \\
&+3 \text { WAVG_SPREADERR_MODEL_I })>0.005) \\
&+((\text { WAVG_SPREAD_MODEL_I } \\
&+ \text { WAVG_SPREADERR_MODEL_I })>0.003) \\
&+((\text { WAVG_SPREAD_MODEL_I } \\
&- \text { WAVG_SPREADERR_MODEL_I })>0.001) .
\end{aligned}
$$

Both NGMIX_CLASS and WAVG_CLASS can have values of 0 , 1,2 , or 3 , with 0 being most star-like and 3 being most galaxylike. Our final stellar sample used NGMIX_CLASS $\leqslant 1$ when the composite-model fit succeeded and WAVG_CLASS $\leqslant 1$ otherwise:

$$
\text { STARS }=\left\{\begin{array}{cl}
\text { NGMIX_CLASS } \leqslant 1, & \text { if ngmix fit succeeds } \\
\text { WAVG_CLASS } \leqslant 1, & \text { otherwise. }
\end{array}\right.
$$

Our stellar selection was designed to yield a compromise between completeness and purity in the resulting stellar sample. In Figure 1 we compare our stellar classification with deeper imaging data from Hyper Suprime-Cam DR1 (Aihara et al. 2018). We find that our selection is $>90 \%$ complete for $g=23.5$ with a galaxy contamination rising from $\lesssim 5 \%$ at $g \leqslant 22.5$ to $\sim 30 \%$ by $g \sim 23.5$. Throughout the paper we refer to the objects passing the selection in Equation (3) as stars.

We constrained our stellar sample to the range $16<$ $g<23.5$. The bright-end limit was imposed to avoid saturation effects from bright stars, while at the faint end we seek to avoid spurious density fluctuations resulting from inhomogeneous survey depth and galaxy contamination. Since we are primarily interested in main-sequence (MS) and red giant branch (RGB) stars associated with old, metal-poor stellar populations, we constrain our sample to the color range $0.0<g-r<1.0$.

In contrast to previous DES photometric calibration techniques (Drlica-Wagner et al. 2015, 2018), no stellar locus regression adjustment was applied to the DES Y3A2 zeropoints derived by the FGCM. Instead, we followed the procedure described in DES Collaboration (2018) to account for interstellar dust extinction. We started with $E(B-V)$ values from the reddening map of Schlegel et al. (SFD; 1998). We computed fiducial interstellar extinction coefficients, $R_{b}$, for each band so that the corrections to the top-of-the-atmosphere 


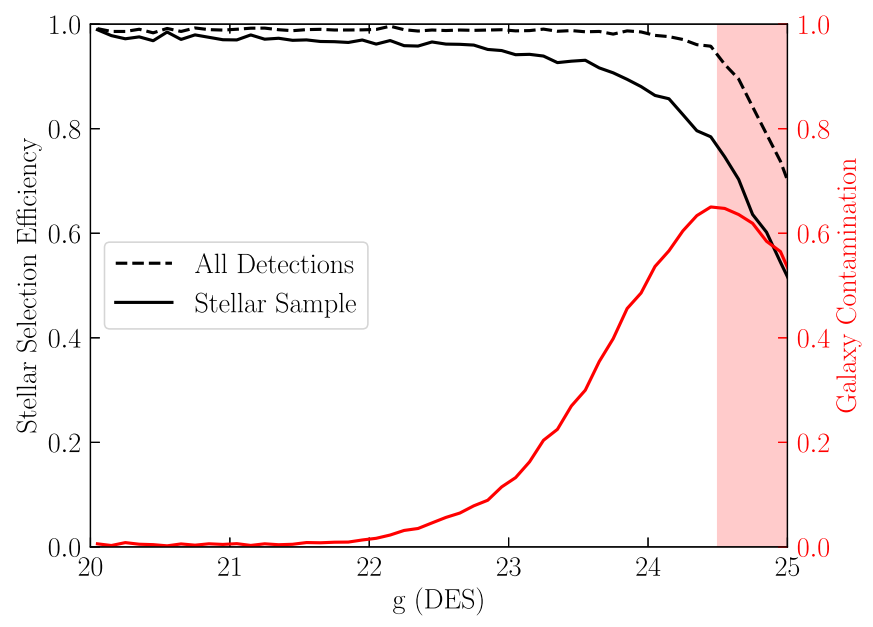

Figure 1. Stellar selection efficiency and galaxy contamination for the DES Y3 data evaluated from a comparison to HSC DR1 using $\sim 18 \mathrm{deg}^{2}$ of overlap in the SDSS Stripe 82 region. The DES efficiency is evaluated with respect to a stellar sample from HSC. The red shaded band indicates the faint magnitude range where the HSC data are affected by star-galaxy confusion and may be less reliable as a test sample. Our stellar classification primarily uses the ngmix multiepoch fitting size parameter and error.

calibrated source magnitudes are $A_{b}=E(B-V) \times R_{b}$. Fiducial coefficients are derived using the Fitzpatrick (1999) reddening law with $R_{V}=3.1$ and the Schlafly \& Finkbeiner (2011) adjusted reddening normalization parameter, $N=0.78$. We integrated over the DES standard bandpasses considering a fixed source spectrum that is constant in spectral flux density per unit wavelength. The resulting multiplicative coefficients for the $g$ and $r$ bands are $R_{g}=3.185$ and $R_{r}=2.140 .^{53}$ Throughout this paper, all magnitudes refer to extinctioncorrected PSF magnitudes derived by ngmix.

We build a high-resolution map of the DES survey coverage to account for missing survey coverage at the boundary of the footprint and gaps associated with saturated stars, bleed trails, and other instrumental signatures. We follow the procedure described in Drlica-Wagner et al. (2018) to transform a vectorized representation of the survey coverage calculated by mangle (Hamilton \& Tegmark 2004; Swanson et al. 2008) into a HEALPix (Górski et al. 2005) coverage fraction map. In each nside $=4096\left(\sim 0.74 \operatorname{arcmin}^{2}\right)$ HEALPix pixel, we oversample the mangle map by a factor of 64 to quantify the simultaneous coverage in the griz bands. The HEALPix nested pixelization scheme makes it trivial to degrade the resolution of this coverage fraction map by summing the coverage fraction of all high-resolution pixels nested within a lower-resolution pixel. We restrict our stream search to regions where the griz detection fraction is greater than $50 \%$, resulting in a total solid angle of $4946 \mathrm{deg}^{2}$. Throughout this paper, all coordinates refer to $\mathrm{J} 2000$ epoch.

\section{Analysis}

\subsection{Matched-filter Selection}

We searched for stellar streams using an unweighted matched-filter algorithm in color-magnitude space (Rockosi et al. 2002; Grillmair 2006; Bonaca et al. 2012; Jethwa et al. 2017). Our matched filter is based on the synthetic

\footnotetext{
53 An update to the DECam standard bandpasses changed these coefficients by $<1$ part per 1000 for the DR1 release (DES Collaboration 2018).
}

isochrone of an old ( $\tau=13 \mathrm{Gyr})$, metal-poor $(Z=0.0002$, $[\mathrm{Fe} / \mathrm{H}]=-1.9)$, stellar population as constructed by Dotter et al. (2008) and implemented in ugali (Bechtol et al. 2015; Drlica-Wagner et al. 2015). We selected stars within a range of colors around the isochrone according to the criteria

$$
\begin{aligned}
(g-r)_{\text {iso }}+ & E \times \operatorname{err}\left(g_{\text {iso }}+\mu+\Delta \mu / 2\right)-C_{1} \\
& <(g-r)< \\
(g-r)_{\text {iso }}+ & E \times \operatorname{err}\left(g_{\text {iso }}+\mu-\Delta \mu / 2\right)+C_{2},
\end{aligned}
$$

where $(g-r)_{\text {iso }}$ represents the predicted color from the synthetic isochrone at a distance modulus of $\mu=m-M$. We parameterize the magnitude-dependent spread in color due to measurement uncertainties as

$$
\operatorname{err}(g)=0.001+e^{(g-27.09) / 1.09}
$$

where the normalization coefficients were derived from fitting the median photometric error as a function of magnitude in the $g$ band (PSF_MAG_G vs. PSF_MAG_ERR_G). We parameterize the selection region around the isochrone with a symmetric magnitude broadening, $\Delta \mu$, an asymmetric color broadening, $C_{1,2}$, and a multiplicative factor for broadening based on photometric uncertainty, $E$. We set the values $\Delta \mu=0.5$, $C_{1,2}=(0.05,0.10)$, and $E=2$ by comparing to the colormagnitude diagrams (CMDs) of old, metal-poor globular clusters and dwarf galaxies (Figure 2).

In Figure 2 we show our matched-filter selection overplotted on binned CMDs from the globular clusters NGC 1260 and NGC 7089 (M2), the ultrafaint dwarf galaxy Reticulum II, and the classical dwarf galaxy Sculptor. Our selection retains $\gtrsim 90 \%$ of stellar sources fainter than the MSTO in an annulus of $0.07<r<0^{\circ} .12$ around NGC 1260 and NGC 7089. This is a conservative estimate of the true efficiency of our selection, since it does not account for contamination from Milky Way foreground stars within this annulus.

In our initial search for stellar streams in Y3A2, we applied our matched-filter isochrone selection over a grid of distance moduli from $14<m-M<19$ spaced at intervals of $\Delta(m-M)=0.3$. This spacing between distance modulus steps was chosen so that sequential isochrone selections overlap by $\gtrsim 75 \%$ to ensure that streams at intermediate distance moduli were detectable (Grillmair 2017). For each distance modulus, we binned stars passing our matched-filter selection into equal-area HEALPix pixels with area of $\sim 0.013 \mathrm{deg}^{2}$ (nside $=512$ ). We divided the number of stars selected in each HEALPix pixel by a map of the coverage fraction at equivalent resolution to produce a coveragecorrected map of stellar density. We smoothed the density maps with a 2D Gaussian symmetric beam with $\sigma=0.3 .^{54} \mathrm{We}$ later repeated this procedure at spacings of $\Delta(m-M)=0.1$ and confirmed that all stream candidates were still detected. We show the smoothed density map after the isochrone selection in Figure 3.

Matched filters are often weighted by the ratio of the density of stars in color-magnitude space between a target stellar population and the background population (e.g., Rockosi et al. 2002). However, this weighting disrupts the Poisson-distributed nature of the counts and does not account for changes in the foreground and background stellar populations across the

\footnotetext{
$\overline{54}$ http://healpy.readthedocs.io/en/latest/generated/healpy.sphtfunc. smoothing.html
} 

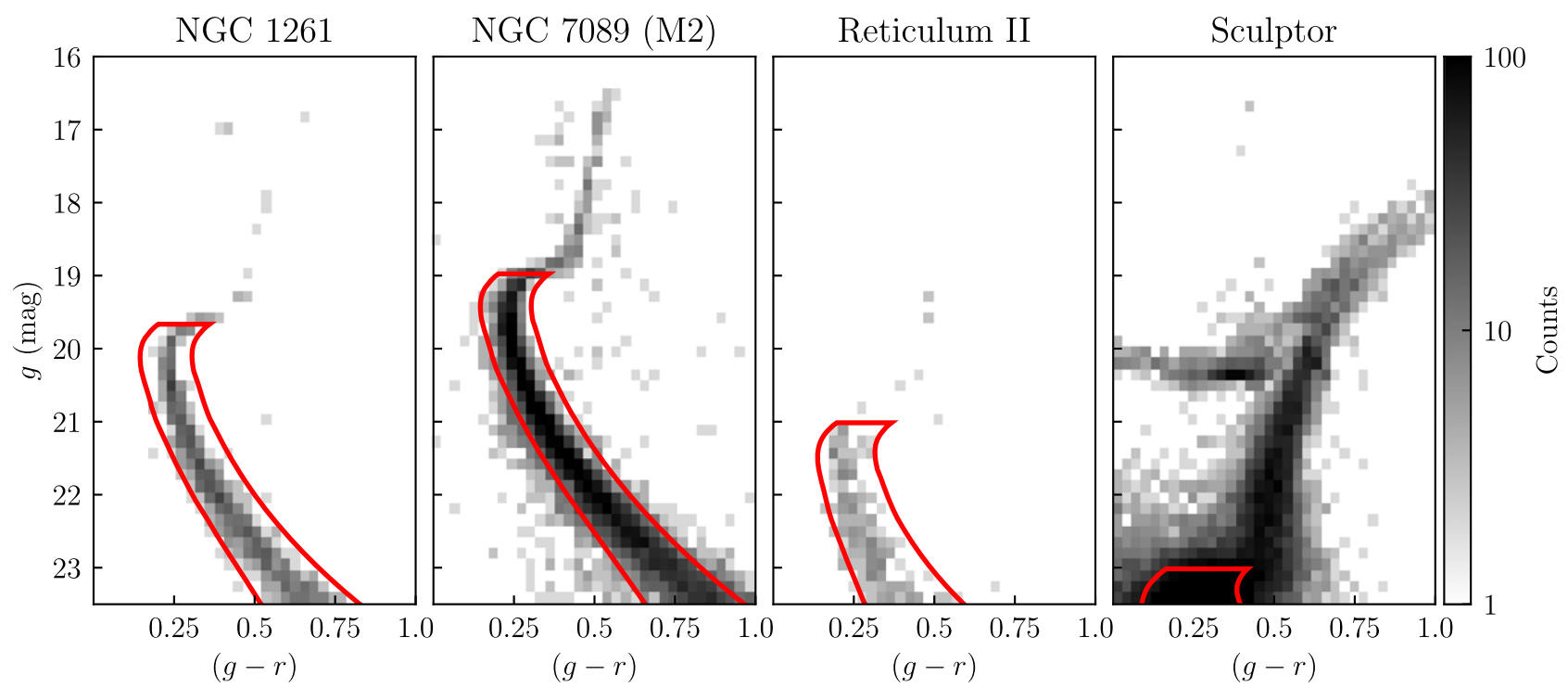

Figure 2. Binned CMD of DES Y3A2 stellar sources selected around the globular clusters NGC 1261 and NGC 7089 (M2), the ultrafaint dwarf galaxy Reticulum II, and the classical dwarf galaxy Sculptor. For the two globular clusters, data are selected in an annulus with $0.07<r<0$. 12 . Stellar sources are selected within $r<0.15$ of Reticulum II and $r<0.20$ of Sculptor. The matched-filter selection region for our stellar stream search is shown with the red outlines.

footprint (e.g., close to the Galactic disk, LMC, or Sagittarius stream). For these reasons, we perform an unweighted matched-filter search, where stars are simply accepted or rejected based on their location in color-magnitude space (e.g., Erkal et al. 2017). As a validation, we repeated our search following the procedure of Rockosi et al. (2002) to build a matched-filter isochrone from the CMDs of the globular cluster NGC 7089 and the average Milky Way foreground population. We apply an additive factor of $\Delta(m-M)$ to shift the CMD of NGC 7089 in distance modulus and thus select for stellar populations over a range of distances. We find that this procedure yields very similar results to our primary unweighted synthetic isochrone selection technique, and all stream candidates reported here were detected by both analyses.

\subsection{Stream Detection}

The Sagittarius $\left(l, b \sim 160^{\circ},-60^{\circ}\right)$, ATLAS $\left(l, b \sim-130^{\circ}\right.$, $\left.-80^{\circ}\right)$, Phoenix $\left(l, b \sim-70^{\circ},-65^{\circ}\right)$, and Tucana III $(l, b \sim$ $\left.-45^{\circ},-55^{\circ}\right)$ streams are clearly visible in smoothed density maps (Figure 3). To further increase our sensitivity to faint stellar streams, we created a smooth model for the stellar foreground and misclassified background galaxies. We mask the dense stellar regions around the Sagittarius stream, the LMC, and the Galactic plane (Figure 4) and fit a 2D, fifth-order polynomial to the distribution of smoothed stellar counts. We subtracted this model from the stellar density to create smooth maps of the residual stellar density (Figure 4). Visual inspection of the foreground-subtracted residual density maps served as the primary technique for identifying new streams.

To facilitate the detection of faint streams, we repeated the procedure described above to generate residual stellar density maps for a range of isochrone selections with distance moduli between $14 \leqslant m-M \leqslant 19$ in steps of $\Delta(m-M)=0.3$. We assembled these sequences of residual density maps into animations of the isochrone-selected stellar density as a function of heliocentric distance. We required that stellar stream candidates appear in the residual density maps for at least two sequential distance moduli. The animations associated with Figures 3 and 4 contain a wealth of information about stellar structure in the Milky Way halo. In this paper we specifically focus on the the most prominent stellar streams, leaving other studies of the outer halo to future work.

We perform our visual search by assembling residual density maps of the full DES footprint and in smaller subregions, which we call "quadrants." Candidates identified in the residual density maps were further examined in color-magnitude space for evidence of a stellar population distinct from the Milky Way foreground (Section 3.3). Only candidates that showed a distinct stellar locus consistent with an old, metal-poor isochrone were included in our list of stellar stream candidates. This search resulted in the detection of the four previously known narrow stellar streams (ATLAS, Phoenix, Tucana III, and Molonglo) and 11 new stream candidates. We report the measured and derived parameters of our stream candidates in Tables 1 and 2, and we discuss each candidate in more detail in Section 4.

\subsection{Stream Characterization}

After candidates are identified in the residual density images, we perform an iterative process to fit the characteristics of each stream:

1. Define stream endpoints from the residual density maps.

2. Fit the transverse stream width to define an "on-stream" region.

3. Fit isochrone parameters to the CMD of on-stream minus off-stream stars.

4. Refit the transverse stream width using the best-fit isochrone selection.

We describe each of these steps in more detail below.

We defined the endpoints of each stream from the residual stellar density map. The residual stellar density map has a pixel scale of $\sim 0$. 1 (nside $=512$ ) and is smoothed by a Gaussian kernel with standard deviation $\sigma=0$. 3. For narrow, prominent streams the endpoints can be measured with an accuracy of better than 0.1 ; however, for fainter and/or more diffuse 

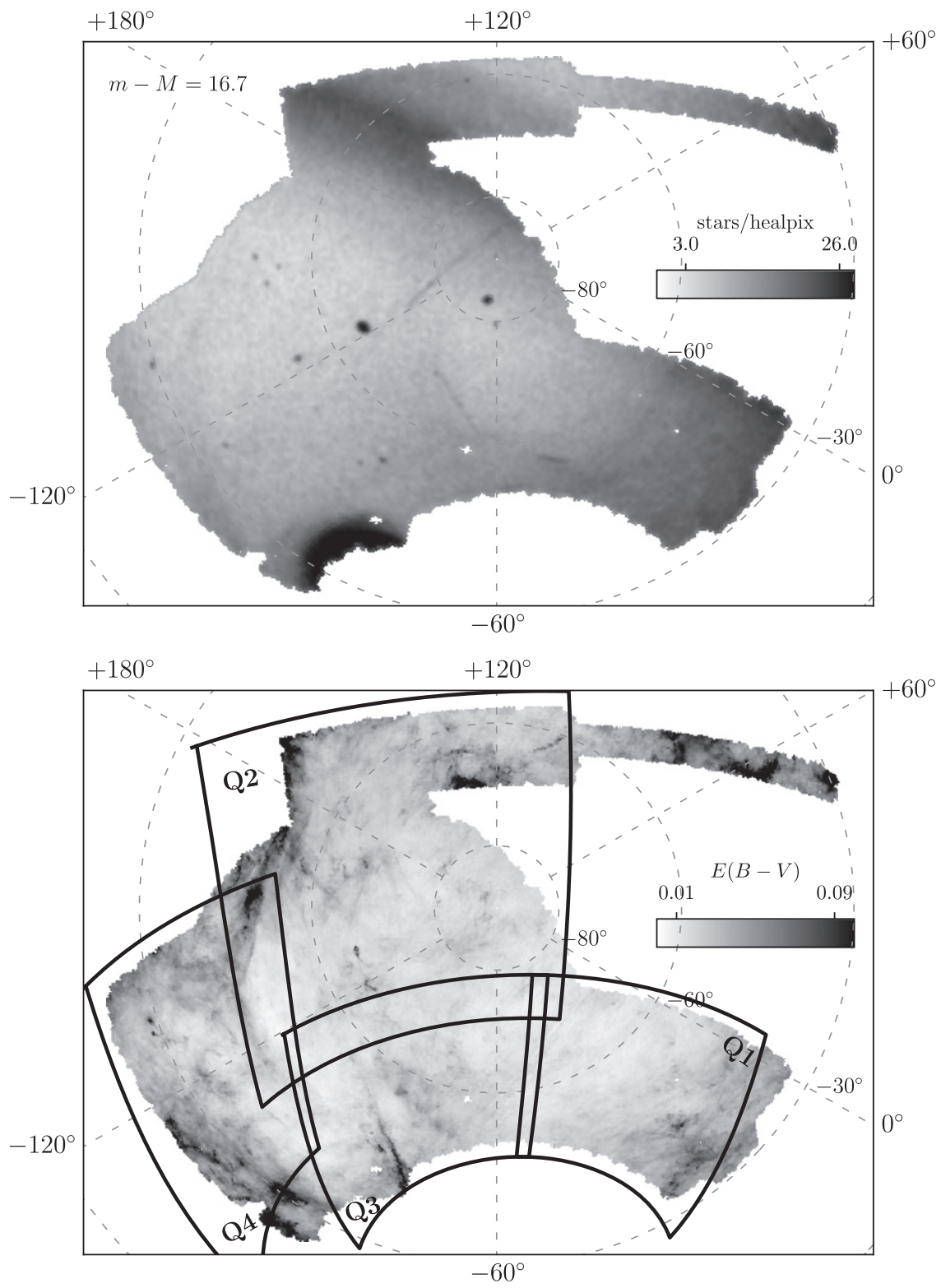

Figure 3. Top: density of stars passing the matched-filter isochrone selection at a distance modulus of $m-M=16.7$. Stars are pixelized into equal-area HEALPix pixels with area of $\sim 0.013 \mathrm{deg}^{2}$ (nside $=512$ ). Contributions from the LMC (lower left), Sagittarius stream (top center), and Galactic thick disk (lower right) can be clearly seen. Bottom: interstellar extinction, $E(B-V)$, estimated by Schlegel et al. (1998). Outlines of the four DES "quadrants" defined in Section 4 are overplotted. Both panels are plotted in Galactic coordinates using a polar Lambert equal-area projection. The animation shows the variation in the density of stars as a function of distance modulus, from 14 to 19 mag.

(An animation of this figure is available.)

structures, measuring endpoints becomes more uncertain. The stream length reported in Table 1 was calculated as the angular separation between the endpoints assuming that the streams follow a great circle on the sky.

For each stream, we calculate the pole of a great circle passing through the endpoints and rotate into a coordinate system where the fundamental plane is aligned with the long axis of the stream. Following the convention of Majewski et al. (2003), we define (heliocentric) longitudinal and latitudinal coordinates $(\Lambda, B)$ for the rotated coordinate system associated with each stream.

As an initial estimate for the width of each stream, we rotate the HEALPix pixels of our raw and residual density maps into the frame of each stream. We then sum the content of
HEALPix pixels along the transverse stream dimension to provide the transverse stream profile. We fit the transverse stream profile with a linear foreground component and a Gaussian stream model with free normalization and standard deviation. The standard deviation of the best-fit Gaussian was taken as an estimate of the stream width, and was used to define signal and background regions for the color-magnitude analysis in the following section. We repeated this procedure after selecting stars consistent with the best-fit isochrone to derive the final stream width.

Stream candidates were examined in color-magnitude space to confirm the presence of a distinct stellar population matched to an old, metal-poor isochrone. An "on-stream" region was selected along the great circle connecting the stream endpoints. 

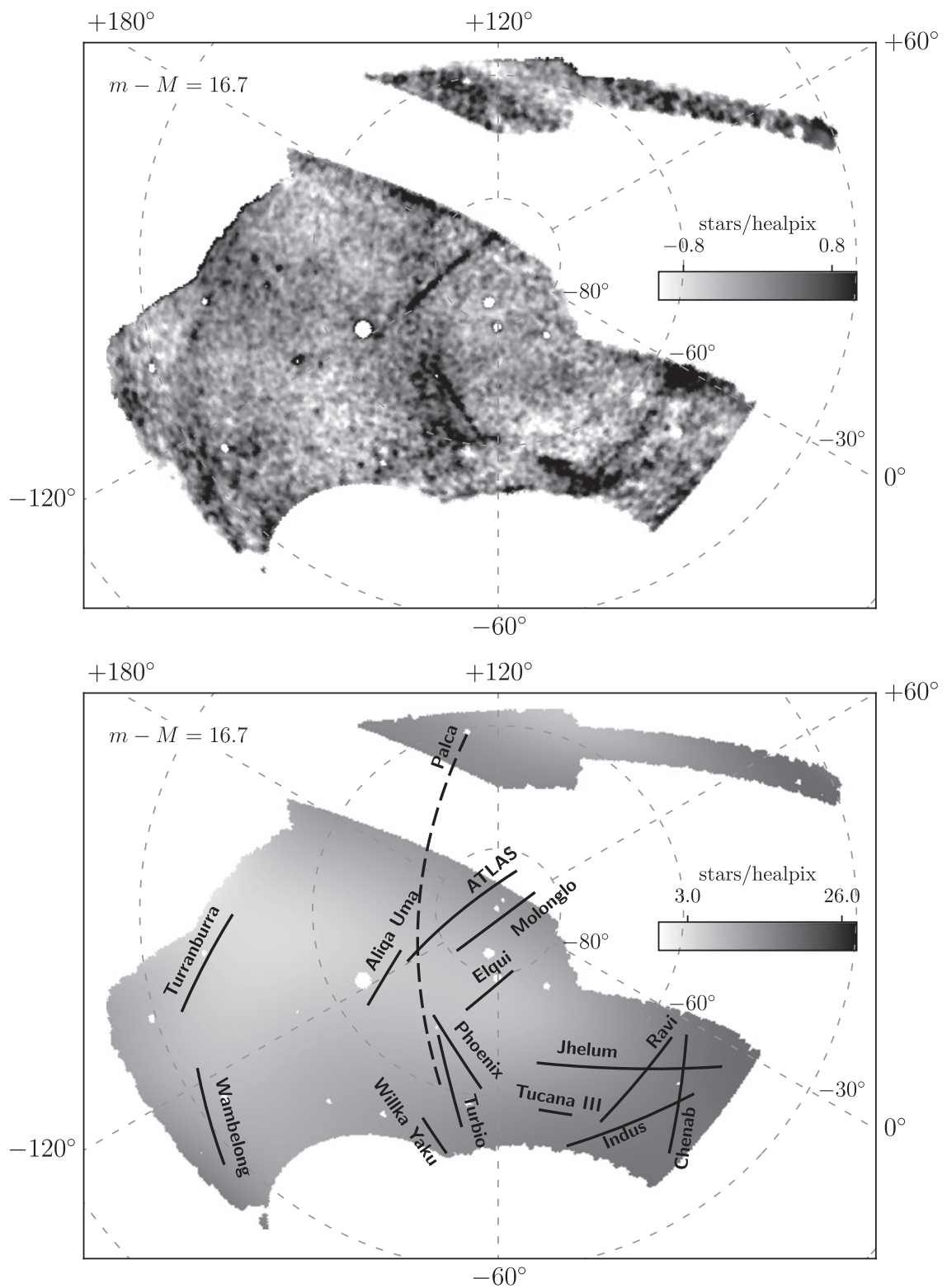

Figure 4. Top: residual density of stars passing the matched-filter isochrone selection at a distance modulus of $m-M=16.7$. Regions around the LMC (lower left), Sagittarius stream (top center), and the Galactic disk (lower right) have been masked to improve the quality of the polynomial background fit. In addition, small regions $\left(r<1^{\circ}\right)$ around bright Milky Way globular clusters, satellite galaxies, and Local Group galaxies have been masked. Bottom: smooth background fit to the stellar density using a 2D, fifth-order polynomial (gray scale). Stellar streams are labeled and overplotted. Palca and ATLAS are traced by a second-order polynomial (Sections 4.2.1 and 4.5, respectively), while other streams are traced by great-circle arcs. Both panels are plotted in Galactic coordinates using a polar Lambert equalarea projection. The animation shows the variation of the residual density of stars and the background fit as a function of distance modulus, from 14 to 19 mag.

(An animation of this figure is available.)

For most streams, this region had a width of $\pm 2 w$, where $w$ is the stream width derived from the standard deviation of the best-fit Gaussian. Two "off-stream" regions were selected with the same shape as the on-stream region, but offset perpendicular to the stream axis by $\pm 4 w$. In some cases this on-stream and off-stream geometry was impossible owing to the boundary of the survey or the presence of large resolved stellar populations (other streams, large dwarf galaxies, etc.). The precise on- and off-stream regions selected for each stream are described in Table 5 in the Appendix. When building on- and off-stream regions, we excise regions around known globular clusters and dwarf galaxies to avoid contaminating the CMD analysis. We calculate the effective solid angle of each region accounting for the excised regions and incomplete survey coverage using the maps described in Section 3.1. We binned the stars in the on-stream region in color-magnitude space with bin size $\Delta g=0.167 \mathrm{mag}$ and $\Delta(g-r)=0.04 \mathrm{mag}$. We calculated the effective foreground contribution in each bin of the CMD using the off-stream regions and correcting the difference in effective solid angle. Hess diagrams were smoothed by a 0.75 pixel Gaussian kernel, and the resulting smooth residual CMD was examined for the presence of a distinct stellar population.

We performed a binned maximum-likelihood fit of the smoothed 2D background-subtracted Hess diagrams using a synthetic isochrone from Dotter et al. (2008) weighted by a 
Table 1

Measured Parameters of Stellar Streams

\begin{tabular}{|c|c|c|c|c|c|c|c|c|c|}
\hline Name & $\begin{array}{c}\text { Endpoints } \\
\left(\alpha_{2000}, \delta_{2000}\right)(\operatorname{deg})\end{array}$ & $\begin{array}{l}\text { Great-circle Pole } \\
\left(\alpha_{2000}, \delta_{2000}\right)(\mathrm{deg})\end{array}$ & $\begin{array}{l}\text { Length } \\
\text { (deg) }\end{array}$ & $\begin{array}{l}\text { Width } \\
\text { (deg) }\end{array}$ & $m-M$ & $\begin{array}{l}\text { Age } \\
(\mathrm{Gyr})\end{array}$ & $Z$ & $N_{*}$ & Significance \\
\hline Tucana III & $(-6.3,-59.7),(3.2,-59.4)$ & $(354.2,30.3)$ & 4.8 & 0.18 & 17.0 & 13.5 & 0.0001 & 700 & 17.0 \\
\hline ATLAS & $(9.3,-20.9),(30.7,-33.2)$ & $(74.3,47.9)$ & 22.6 & 0.24 & 16.8 & 11.0 & 0.0007 & 1600 & 13.9 \\
\hline Molonglo & $(6.4,-24.4),(13.6,-28.1)$ & $(62.3,51.0)$ & 7.4 & 0.32 & 16.8 & 13.5 & 0.0010 & 700 & 5.2 \\
\hline Phoenix & $(20.1,-55.3),(27.9,-42.7)$ & $(311.2,14.0)$ & 13.6 & 0.16 & 16.4 & 13.0 & 0.0004 & 700 & 11.1 \\
\hline Indus & $(-36.3,-50.7),(-8.0,-64.8)$ & $(24.8,21.6)$ & 20.3 & 0.83 & 16.1 & 13.0 & 0.0007 & 9700 & 21.4 \\
\hline Jhelum & $(-38.8,-45.1),(4.7,-51.7)$ & $(359.1,38.2)$ & 29.2 & 1.16 & 15.6 & 12.0 & 0.0009 & 4600 & 18.6 \\
\hline Ravi & $(-25.2,-44.1),(-16.0,-59.7)$ & $(53.2,11.7)$ & 16.6 & 0.72 & 16.8 & 13.5 & 0.0003 & 2300 & 10.3 \\
\hline Chenab & $(-40.7,-59.9),(-28.3,-43.0)$ & $(255.5,14.4)$ & 18.5 & 0.71 & 18.0 & 13.0 & 0.0004 & 1700 & 15.1 \\
\hline Elqui & $(10.7,-36.9),(20.6,-42.4)$ & $(64.0,38.5)$ & 9.4 & 0.54 & 18.5 & 12.0 & 0.0004 & 700 & 18.4 \\
\hline Aliqa Uma & $(31.7,-31.5),(40.6,-38.3)$ & $(94.5,36.7)$ & 10.0 & 0.26 & 17.3 & 13.0 & 0.0004 & 400 & 9.1 \\
\hline Turbio & $(28.0,-61.0),(27.9,-46.0)$ & $(297.8,-0.1)$ & 15.0 & 0.25 & 16.1 & 13.0 & 0.0004 & 1000 & 7.9 \\
\hline Willka Yaku & $(36.1,-64.6),(38.4,-58.3)$ & $(316.0,4.7)$ & 6.4 & 0.21 & 17.7 & 11.0 & 0.0006 & 600 & 7.1 \\
\hline Turranburra & $(59.3,-18.0),(75.2,-26.4)$ & $(123.5,53.3)$ & 16.9 & 0.60 & 17.2 & 13.5 & 0.0003 & 1300 & 14.4 \\
\hline Wambelong & $(90.5,-45.6),(79.3,-34.3)$ & $(328.7,-27.3)$ & 14.2 & 0.40 & 15.9 & 11.0 & 0.0001 & 500 & 5.9 \\
\hline Palca & $(30.3,-53.7),(16.2,2.4)$ & $(286.6,-9.9)$ & 57.3 & $\ldots$ & 17.8 & 13.0 & 0.0004 & $\ldots$ & $\ldots$ \\
\hline
\end{tabular}

Note. Measured characteristics of stellar streams detected in DES Y3A2 data. The first section reports DES measurements of previously known streams, while the second section reports narrow streams discovered by DES. The broad stream/stellar overdensity, Palca, is given its own section. Endpoints and great-circle poles are reported in equatorial coordinates and are derived from the residual stellar density analysis described in Section 3.2. Stream lengths are calculated as the angular separation between endpoints, while stream widths, $w$, come from the standard deviation of a Gaussian fit to the transverse stream profile. Distance moduli, ages, and metallicities were calculated by fitting a Dotter et al. (2008) isochrone to the Hess diagrams described in Section 3.3. The number of stars is calculated by summing MS stars in the background-subtracted Hess diagram. The significance is calculated as the signal-to-noise ratio between the on-stream and off-stream regions.

Chabrier (2001) initial mass function (IMF). We built a binned Poisson likelihood function for the observed number of stars in each CMD bin given the number of stars predicted by our isochrone model convolved by the empirically determined photometric measurement uncertainties (Section 2). We simultaneously fit the richness, distance modulus, age, and metallicity of the isochrone model to the observed excess counts in the Hess diagram. The richness is a normalization parameter representing the total number of stream member stars with mass $>0.1 M_{\odot}$ (Bechtol et al. 2015). For roughly half the stream candidates, the data were unable to reliably constrain all four parameters simultaneously, and we fixed the age $(\tau=13 \mathrm{Gyr})$ and metallicity $(Z=0.0004)$ while fitting richness and distance modulus. Furthermore, we note that there is a significant degeneracy between the age, metallicity, and distance modulus. We estimate a systematic uncertainty on the distance modulus of $\sigma(m-M) \sim 0.4 \mathrm{mag}$, while spectroscopic observations are essential to break the degeneracy between age and metallicity.

Several streams, specifically those closer to the Galactic plane, suffer from over- or undersubtraction due to gradients in the surrounding stellar density. Mis-subtraction will bias estimates of the richness and total luminosity. To mitigate these issues, we estimate the stellar content of each stream based on the number of MS stars within a region around the best-fit isochrone. We apply a narrow isochrone selection based on Equation (4) using the best-fit age, metallicity, and distance modulus (Table 1) and the selection parameters $\Delta \mu=0.5$, $C_{1,2}=(0.05,0.05)$, and $E=1 .{ }^{55} \mathrm{We}$ sum the content of the background-subtracted Hess diagram within this selection region. We then correct the number of stars for the fraction

\footnotetext{
55 We find that this selection is $67 \%$ efficient for stars in the globular cluster NGC 7089 (M2). This efficiency is taken into account when calculating the richness and stellar mass.
}

of the stream width contained in the spatial selection region, to estimate the total number of MS stream stars within the spatial and magnitude range of DES. We record this value as $N_{*}$ in Table 1. We then use the isochrone model along with a Chabrier (2001) IMF to estimate the total stellar mass, luminosity, and absolute magnitude in Table 2.

We refit the stream width after applying an isochrone selection consistent with the best-fit isochrone. We also calculate the statistical significance of each stream from the on-stream and area-corrected off-stream regions, $S \equiv$

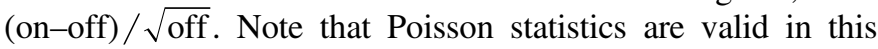
case owing to our use of an unweighted matched filter. For consistency, all on-stream regions had a width of $\pm w$ for this calculation. We report the measured parameters of each stream in Table 1 and derived physical parameters in Table 2. In addition, we use this absolute magnitude to calculate an average surface brightness, which we estimate assuming that $68 \%$ of the luminosity is contained within $\pm w$ of the stream axis.

In Table 2 we also provide estimates of the progenitor masses. We use the relation between the stream width and the progenitor mass derived in Erkal et al. (2016b). More precisely, we use Equation (27) from Erkal et al. (2016a), where the progenitor mass is given in terms of the stream width as viewed from the Galactic center and the enclosed mass of the Milky Way at the stream's location. For the mass of the Milky Way, we use the best-fit model in McMillan (2017), who used a range of data to constrain the Milky Way potential. We then use GALPOT (Dehnen \& Binney 1998) to evaluate the circular velocity (and hence the enclosed mass) as a function of galactocentric radius. We note that this method assumes that the streams are on a circular orbit and only works on average for streams on eccentric orbits (Erkal et al. 2016b). Furthermore, this method also assumes that the streams have not 
Table 2

Derived Parameters of Stellar Streams

\begin{tabular}{|c|c|c|c|c|c|c|c|}
\hline Name & $\begin{array}{l}\text { Distance } \\
\quad(\mathrm{kpc})\end{array}$ & $\begin{array}{l}\text { Length } \\
(\mathrm{kpc})\end{array}$ & $\begin{array}{l}\text { Width } \\
\text { (pc) }\end{array}$ & $\begin{array}{l}\text { Stellar Mass } \\
\qquad\left(10^{3} M_{\odot}\right)\end{array}$ & $\begin{array}{c}M_{V} \\
\text { (mag) }\end{array}$ & $\begin{array}{c}\mu_{V} \\
\left(\text { mag } \operatorname{arcsec}^{-2}\right)\end{array}$ & $\begin{array}{l}\text { Progenitor Mass } \\
\qquad\left(10^{4} M_{\odot}\right)\end{array}$ \\
\hline Tucana III & 25.1 & 2.1 & 79 & 3.8 & -3.8 & 32.0 & 8 \\
\hline ATLAS & 22.9 & 9.0 & 96 & 7.4 & -4.5 & 33.0 & 12 \\
\hline Phoenix & 19.1 & 4.5 & 53 & 2.8 & -3.6 & 32.6 & 3 \\
\hline Indus & 16.6 & 5.9 & 240 & 34.0 & -6.2 & 31.9 & 650 \\
\hline Chenab & 39.8 & 12.9 & 493 & 18.3 & -5.7 & 34.1 & 780 \\
\hline Elqui & 50.1 & 8.2 & 472 & 10.4 & -4.9 & 34.3 & 320 \\
\hline Aliqa Uma & 28.8 & 5.0 & 131 & 2.3 & -3.4 & 33.8 & 18 \\
\hline Turbio & 16.6 & 4.3 & 72 & 3.5 & -3.9 & 32.6 & 10 \\
\hline Willka Yaku & 34.7 & 3.9 & 127 & 4.6 & -4.1 & 32.9 & 14 \\
\hline Turranburra & 27.5 & 8.1 & 288 & 7.6 & -4.7 & 34.0 & 180 \\
\hline
\end{tabular}

Note. Derived physical parameters of stellar streams detected in DES Y3A2 data. Heliocentric distances are calculated by fitting a Dotter et al. (2008) isochrone to the Hess diagrams described in Section 3.3 and have an error of $\sim 20 \%$. Other physical parameters are derived from the measured parameters in Table 1 assuming this distance. Stream lengths are calculated from the angular distance between the stream endpoints, while the widths represent the standard deviation of the best-fit Gaussian. Stellar masses, absolute magnitudes, and surface brightnesses are derived from the richness of the best-fit isochrone model assuming a Chabrier (2001) IMF. The absolute magnitude is derived from the DES $g$ and $r$ bands following the prescription of Bechtol et al. (2015). The surface brightness is derived assuming that $68 \%$ of the luminosity is contained within the stream width of $\pm w$. The progenitor masses are estimated using the results of Erkal et al. (2016b).

Table 3

Galactocentric Parameters of Stellar Streams

\begin{tabular}{|c|c|c|c|c|}
\hline Name & $\begin{array}{c}x_{1}, y_{1}, z_{1} \\
(\mathrm{kpc})\end{array}$ & $\begin{array}{c}x_{2}, y_{2}, z_{2} \\
\quad(\mathrm{kpc})\end{array}$ & $\begin{array}{l}\overline{R_{\mathrm{GC}}} \\
(\mathrm{kpc})\end{array}$ & $\begin{array}{c}(\phi, \psi) \\
(\mathrm{deg})\end{array}$ \\
\hline Tucana III & $(2.7,-9.4,-20.5)$ & $(0.8,-10.2,-21.1)$ & 23 & $(285.9,64.6)$ \\
\hline ATLAS & $(-8.5,2.8,-22.7)$ & $(-11.7,-5.6,-22.0)$ & 25 & $(157.3,68.5)$ \\
\hline Phoenix & $(-4.5,-8.3,-16.7)$ & $(-8.4,-6.4,-17.9)$ & 20 & $(235.4,60.6)$ \\
\hline Indus & $(2.9,-2.6,-12.0)$ & $(-0.6,-7.3,-12.7)$ & 14 & $(321.3,72.0)$ \\
\hline Chenab & $(18.9,-12.5,-26.3)$ & $(15.5,-1.5,-31.9)$ & 35 & $(28.7,68.0)$ \\
\hline Elqui & $(-2.4,-6.3,-49.4)$ & $(-5.2,-13.9,-48.0)$ & 50 & $(159.6,89.9)$ \\
\hline Aliqa Uma & $(-13.4,-6.7,-27.6)$ & $(-13.5,-11.4,-26.0)$ & 31 & $(171.2,66.0)$ \\
\hline Turbio & $(-5.0,-9.0,-13.5)$ & $(-7.8,-6.3,-15.4)$ & 18 & $(208.7,57.3)$ \\
\hline Willka Yaku & $(-1.5,-21.4,-26.4)$ & $(-4.7,-20.0,-28.1)$ & 34 & $(229.7,56.9)$ \\
\hline Turranburra & $(-24.6,-9.8,-19.9)$ & $(-23.6,-16.6,-15.8)$ & 33 & $(155.3,47.5)$ \\
\hline
\end{tabular}

Note. Galactocentric parameters of stellar streams detected in DES Y3A2 data. Transformation into galactocentric coordinates is performed assuming that the Earth resides at $(8.3 \mathrm{kpc}, 0,0)$. The galactocentric azimuthal and polar angles, $(\phi, \psi)$, are defined as in Figure 1 of Erkal et al. (2016b). Both endpoints are assumed to be at the heliocentric distance quoted in Table 1.

fanned out significantly as a result of being in a nonspherical potential (Pearson et al. 2015; Erkal et al. 2016b). As such, this method should be seen as giving a rough estimate of the progenitor mass.

In Table 3 we present stream parameters in galactocentric coordinates, assuming that the Sun is located $8.3 \mathrm{kpc}$ from the Galactic center (Gillessen et al. 2009; de Grijs \& Bono 2016). Galactocentric Cartesian coordinates are provided for endpoints of each stream assuming the heliocentric distance derived in Table 2 . We provide longitude and co-latitude $(\phi, \psi)$ for the pole of a galactocentric orbit passing through the endpoints of each stream. ${ }^{56}$ Assuming a single heliocentric distance for each stream naturally introduces a gradient in the galactocentric radius. We use the average galactocentric radius when calculating galactocentric great-circle orbits. These galactocentric parameters are primarily used to identify potential associations in Section 5.1.

\footnotetext{
${ }^{56}$ Our definition of $\phi$ and $\psi$ conforms to Figure 1 of Erkal et al. (2016b).
} 


\section{Stellar Stream Candidates}

As part of our search for stellar streams, we divided the DES footprint into four "quadrants" (Q1-Q4). These quadrants were designed to be large enough to fully contain streams spanning $>20^{\circ}$, while providing a more detailed view than maps of the full footprint could offer. These quadrants offer a useful unit to subdivide the DES stellar stream candidates, and we discuss each quadrant in turn. We choose to name our stellar stream candidates after aquatic terms used by the geographically distinct cultures of India (Q1), Chile (Q2 and Q3), and Australia (Q4).

\subsection{First Quadrant ${ }^{57}$}

The first quadrant (Q1) covers the western portion of the DES footprint from $-45^{\circ} \lesssim \alpha_{2000} \lesssim 10^{\circ}$ and $-65^{\circ} \lesssim \delta_{2000} \lesssim$ $-40^{\circ}$ (Figure 5). While the DES data extend to $\alpha_{2000} \gtrsim-55^{\circ}$, the low-order polynomial background fit has difficulty modeling the rapidly varying stellar density at these lower Galactic latitudes. Q1 includes the Tucana III satellite and stream, the two most prominent new stellar streams, Indus and Jhelum, and two lower significance streams, Chenab and Ravi. In addition, diffuse stellar overdensities are found at the northern $\left(\alpha_{2000}, \delta_{2000} \sim-28^{\circ},-42^{\circ}\right)$ and southern $\left(\alpha_{2000}\right.$, $\delta_{2000} \sim-10^{\circ},-63^{\circ}$ ) edges of $\mathrm{Q} 1$; however, the footprint boundary makes it difficult to perform a quantitative evaluation of these structures.

\subsubsection{Tucana III Stream}

The Tucana III stellar stream is located at a distance of $\sim 25 \mathrm{kpc}$, extending at least $\pm 2^{\circ}$ from the ultrafaint satellite Tucana III (Drlica-Wagner et al. 2015). We find that Tucana III appears prominently in the Q1 residual density maps with a projected length of $5^{\circ}$ extending from $\left(-6.3,-59^{\circ} .7\right)$ to $\left(3^{\circ} .2,-59^{\circ} .4\right)$ (Figure 5).

In Figure 6, we show a Hess diagram calculated by subtracting a local background estimate derived from offstream regions on either side of the Tucana III stream. Despite a well-defined MS and visible RGB, our likelihood analysis has trouble simultaneously fitting the richness, distance modulus, age, and metallicity of the Tucana III stream. Assuming a distance modulus of $m-M=17.0$ and a metallicity of $Z=0.0001([\mathrm{Fe} / \mathrm{H}]=-2.24)$ from Drlica-Wagner et al. (2015), we find that the MSTO of Tucana III is well described by an age of $\tau=13.5 \mathrm{Gyr}$. This is older than the $\tau=10.9 \mathrm{Gyr}$ reported by Drlica-Wagner et al. (2015), which is due in part to a correction to the synthetic isochrones using an updated version of the DECam filter throughput (T. S. Li et al. 2018, in preparation). In addition, the change in photometric calibration between the DES Y2Q1 and Y3A2 data sets is found to introduce a small color shift.

Spectroscopic observations have been unable to conclusively classify Tucana III as an ultrafaint galaxy or star cluster (Simon et al. 2017). The unresolved velocity dispersion $\left(\sigma_{v}<1.5 \mathrm{~km} \mathrm{~s}^{-1}\right.$ at $95.5 \%$ confidence) and metallicity spread $\left(\sigma_{[\mathrm{Fe} / \mathrm{H}]}<0.19\right.$ at $95 \%$ confidence $)$ are both low for an ultrafaint dwarf galaxy. However, the mean metallicity $([\mathrm{Fe} / \mathrm{H}]=$ $\left.-2.42_{-0.08}^{+0.07}\right)$ and large physical size $\left(r_{1 / 2}=44 \pm 6 \mathrm{pc}\right)$ are both

\footnotetext{
57 Due to overlap with the constellation Indus, which shares a name with a Pakistani and Indian river, and to honor the tradition of astronomy in the region, new stellar stream candidates in this quadrant are named after rivers in Pakistan and India (including the Indus River itself).
}
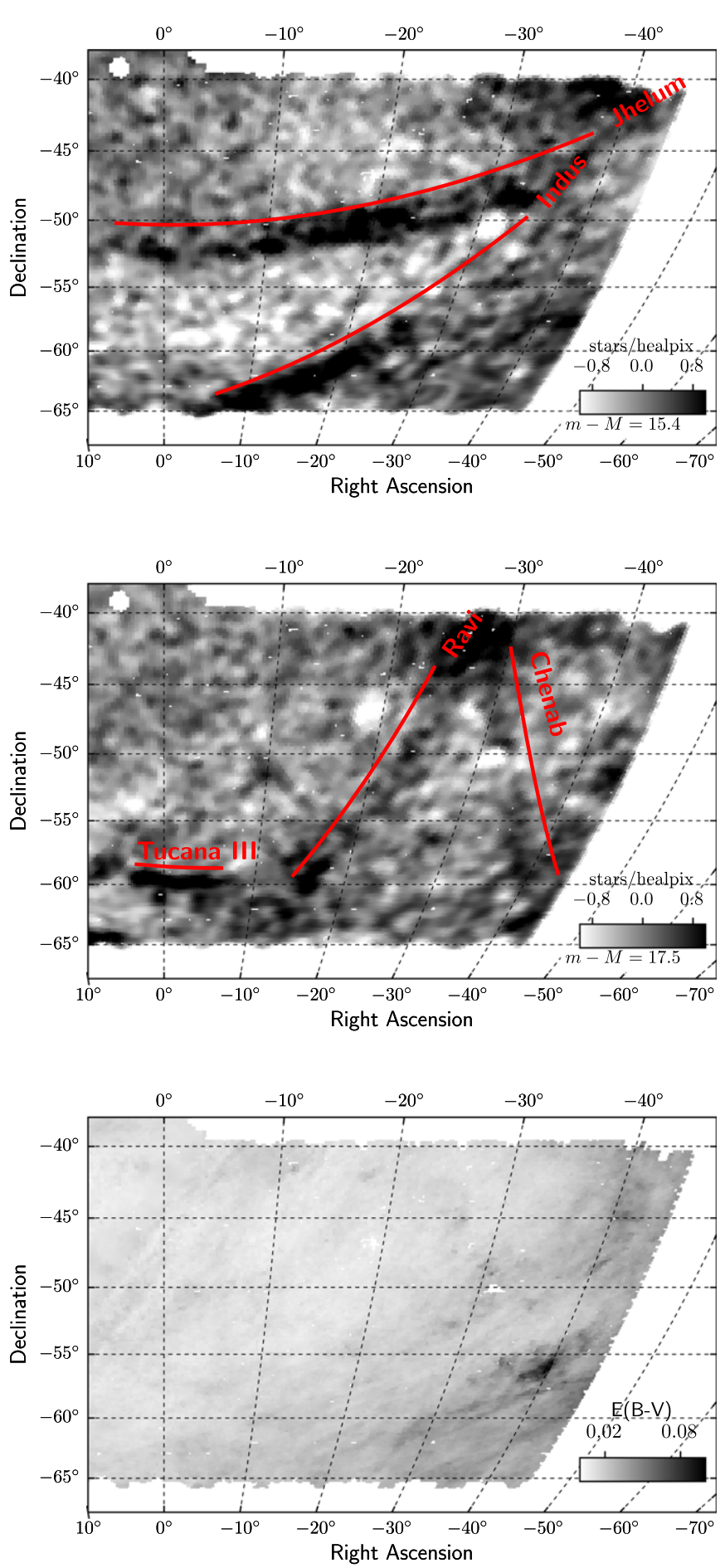

Figure 5. Residual stellar density map in Q1 after subtracting a smooth background model from the distribution of isochrone filtered stars (equal-area McBryde-Thomas flat-polar quartic projection). Streams are marked with great circles that are aligned with the major axis of the stream and offset perpendicularly by 1.5 . Top: isochrone selection with $m-M=16.4$. Middle: isochrone selection with $m-M=17.5$. Bottom: interstellar reddening, $E(B-V)$, from Schlegel et al. (1998). The animation shows the residual stellar density map in Q1 as a function of distance modulus, from 14 to 19 mag.

(An animation of this figure is available.)

unusual for a globular cluster. In addition, Simon et al. (2017) argue that the mass-to-light ratio of the core of Tucana III is larger than that of a globular cluster, $M / L>20 M_{\odot} / L_{\odot}$, based on its proximity to the Galactic center and the nondetection of a 


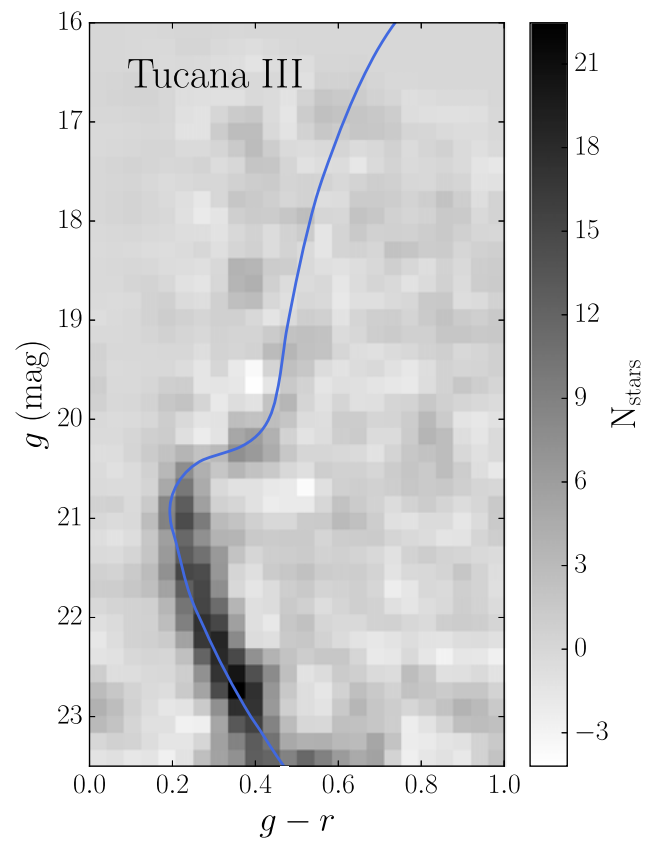

Figure 6. Background-subtracted binned color-magnitude Hess diagram for stars associated with the Tucana III stellar stream. The background is estimated from an off-stream region parallel to the stream and is area corrected and subtracted from the on-stream region. The Hess diagram is smoothed with a 2D Gaussian kernel with a standard deviation of 0.75 pixels. Darkly colored pixels correspond to higher residual stellar density, while lighter pixels represent underdense regions in color-magnitude space. A synthetic isochrone from Dotter et al. (2008) is overplotted with best-fit parameters described in Table 1. The isochrone fitting procedures are described in Sections 3.3 and 4.

velocity gradient out to $90 \mathrm{pc}$. The core of Tucana III lies slightly offset from the luminosity-metallicity relationship for ultrafaint galaxies (Kirby et al. 2013). Simon et al. (2017) note that if Tucana III has been stripped of $\sim 70 \%$ of its stellar mass, then it would lie directly on the metallicity-luminosity relation of ultrafaint dwarfs. We find that the total stellar mass of the Tucana III stream, including the core and the tidal tails, is $3.8 \times 10^{3} M_{\odot}$, which is 4.75 times the stellar mass of the Tucana III core (Drlica-Wagner et al. 2015). This corresponds to a mass loss of $79 \%$, which moves the Tucana III progenitor system onto the luminosity-metallicity relationship for dwarf galaxies.

We search for indications of a distance gradient following a similar procedure to that applied to the Sagittarius stream by Koposov et al. (2012). We transform to a coordinate system oriented along the stream axis and divide the stellar counts into eight longitudinal bins. Within each longitudinal bin, we examine the mean magnitude of MSTO stars satisfying the criteria $0.20<(g-r)<0.24$. We find that the mean magnitude of the MSTO changes from $g \sim 16.75$ at the western end of the stream to $g \sim 17.19$ on the eastern end. Fitting a linear gradient model to these data yields a distance gradient of $0.16 \pm 0.06 \mathrm{mag} \mathrm{deg}^{-1}$. This measurement implies that the Tucana III stream spans $\sim 4 \mathrm{kpc}$ in distance with a total physical extent of $\sim 4.5 \mathrm{kpc}$ and that it is on a radial orbit.

\subsubsection{Indus Stream}

Indus is the first of four new stellar stream candidates detected in Q1. Indus has an angular extent of $20^{\circ} .3$ with a projected width of 0.83 . It may extend beyond the southern edge of the DES footprint in the direction of the SMC; however, at a best-fit distance of $16.6 \mathrm{kpc}(m-M=16.1)$, it is unlikely that there is a physical association between the Indus stream and the Magellanic Clouds (located $\sim 3$ times farther away). The physical width of the Indus stream, $\sigma=240 \mathrm{pc}$ $(\mathrm{FWHM}=565 \mathrm{pc})$, is comparable to that of the Orphan stream (FWHM $=688$ pc; Belokurov et al. 2007b) and considerably larger than known globular cluster streams. There is no obvious progenitor for Indus (Section 5.1); however, its width may indicate that the Indus stream is the disrupted remains of a faint dwarf galaxy.

The MS of the Indus stream is seen prominently in colormagnitude space (Figure 7), with an estimated absolute magnitude of $M_{V}=-6.2$ (Table 2). The measured metallicity of the Indus stream, $Z=0.0007([\mathrm{Fe} / \mathrm{H}]=-1.4)$, is considerably higher than would be expected for a dwarf galaxy with similar luminosity (Kirby et al. 2013). The proximity of the Galactic bulge makes it difficult to model the stellar foreground in the vicinity of Indus, and it is possible that foreground contamination in the RGB of Indus may be artificially inflating the measured metallicity (an even more pronounced example can be seen in Jhelum).

The southern portion of the Indus stream becomes confused with a more distant diffuse stellar structure $(m-M \geqslant 16.5)$ that extends toward the Tucana III stream. Due to the incomplete southern coverage of DES, we cannot determine whether this is the signature of another stream or a diffuse stellar cloud. Other DECam imaging in the regions of the Magellanic Clouds-e.g., the Survey of the Magellanic Stellar History (Nidever et al. 2017) and the Magellanic Satellites Survey (Drlica-Wagner et al. 2016; Pieres et al. 2017, MagLiteS; —-may be able to clarify this question in the near future. However, kinematic information will be necessary to test for any physical connection between Indus/Tucana III and this putative diffuse structure.

\subsubsection{Jhelum Stream}

The Jhelum stream is comparable to Indus in width, $w=1.16$, and due to its orientation on the sky, a longer portion of the stream is contained within the DES footprint $\left(L \sim 29^{\circ} .2\right)$. At a distance of $13.2 \mathrm{kpc}(m-M=15.6)$, Jhelum is closer than Indus; however, both streams can be detected by our isochrone selection simultaneously for distance moduli $15.0 \lesssim m-M \lesssim 16.2$. The average physical width of Jhelum is $267 \mathrm{pc}$, though narrowing is seen at the eastern end of the stream. While Jhelum appears curved in Figure 5, the observed curvature is well matched by a great circle on the sky.

The Hess diagram for the Jhelum stream shows a prominent MS, but it also shows some foreground contamination above the MSTO, as well as some evidence of oversubtraction. In order to reduce contamination, we selected a narrower onstream region of width $\pm w$. Additionally, to reduce the impact of Galactic foreground stars, we first fit the richness, distance modulus, age, and metallicity using just the eastern portion of the stream (higher Galactic latitude). We fix the age and metallicity at the best-fit values from this initial fit and then refit the richness and distance modulus using the full extent of the stream. Similar to Indus, we find a high metallicity, $Z=0.0009$, which is likely influenced by foreground contamination.

The physical similarity and proximity of the Indus and Jhelum streams are suggestive of a possible physical connection between the two streams. To investigate the possibility that Indus and Jhelum may be different orbital arms of the same 

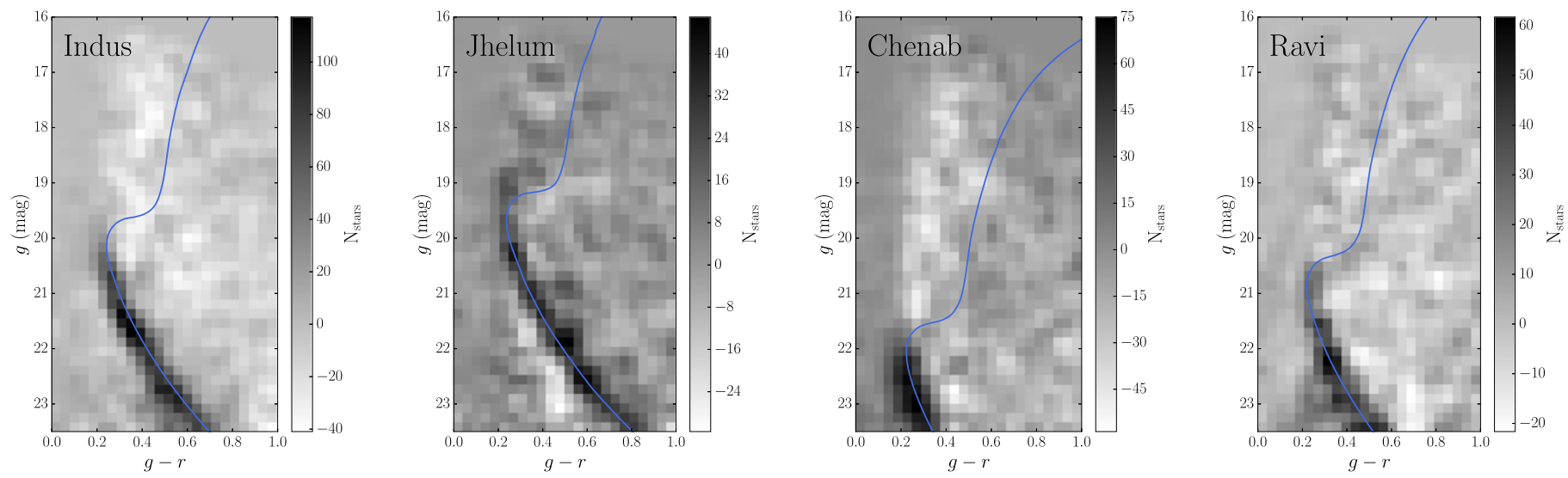

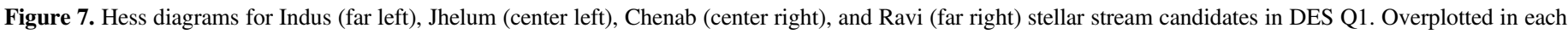
panel is a Dotter et al. (2008) synthetic stellar isochrone with parameters determined from the best fit in Table 1 . These panels are similar to Figure 6.

progenitor, we transformed both into galactocentric coordinates (Table 3). We find that the two streams are at a similar galactocentric radius, $R_{\mathrm{GC}} \sim 11-13 \mathrm{kpc}$, but that the galactocentric great-circle orbits have poles that differ by $\sim 22^{\circ}$. For a flattening $q=0.9$ and an initial polar angle $\psi=75^{\circ}$, the expected precession after one orbit is $\Delta \phi \sim-10^{\circ}$ (Erkal et al. $2016 \mathrm{~b}$ ). This suggests that if Indus and Jhelum are associated with the same progenitor, that progenitor has experienced a more highly asymmetric gravitational potential. Such precession is possible if the progenitor is on an eccentric orbit that takes it close to the Galactic plane, or if the Milky Way halo is more heavily flattened than previously expected. Additional kinematic information is necessary to confirm or refute this hypothesis.

\subsubsection{Ravi Stream}

The Ravi stream candidate is a tenuous feature detected in Q1. It extends from the lower region of the DES footprint up to the Q1 northern overdensity described in Section 4.1.5. The Ravi stream crosses the Tucana II dwarf galaxy in projection; however, a fit to the Hess diagram in Figure 7 puts it at less than half the distance. Due to its higher Galactic latitude and orientation nearly parallel to the Galactic plane, the CMD of Ravi appears considerably cleaner than some of the other streams in this quadrant, with a pronounced MS and less indication of foreground contamination at bright magnitudes. We report the best-fit age, metallicity, and distance modulus in Table 1.

\subsubsection{Chenab Stream}

The Chenab stream candidate runs nearly perpendicular to Indus and Jhelum, but at a significantly larger distance of $39.8 \mathrm{kpc}(m-M=18.0)$. Like the other new streams in this quadrant, Chenab has a large angular size, $0^{\circ} .71$, and a physical width of $\sigma=493 \mathrm{pc}$. The measured extent of Chenab is 18.5 $(12.9 \mathrm{kpc})$. The analysis of the Chenab stream is complicated by contamination from the Milky Way foreground.

Chenab intersects a diffuse stellar overdensity near the northern edge of the DES footprint. This overdensity spans from $-32^{\circ} \lesssim \alpha_{2000} \lesssim-22^{\circ}$ and $-45^{\circ} \lesssim \delta_{2000} \lesssim-40^{\circ}$ and is apparent in the residual density maps selected for isochrones between $17<m-M<19$. It is possible that this overdensity could be an extended spur of the Sagittarius stream, the ridgeline of which passes $\sim 11^{\circ}$ from the northwest corner of the DES footprint at a heliocentric distance of $\sim 25 \mathrm{kpc}$ ( $m-M \sim 17$; Majewski et al. 2004; Law et al. 2005).

\subsection{Second Quadrant ${ }^{58}$}

The second quadrant (Q2) spans $0^{\circ}<\alpha_{2000} \lesssim 60^{\circ}$ and $-42^{\circ}<\delta_{2000}<7^{\circ}$ (Figure 8). This quadrant contains the Sagittarius stream, which we have masked from our analysis in order to increase our sensitivity to fainter new streams. We detect four narrow streams in this region, including the previously known ATLAS (Koposov et al. 2014) stream, a possible extension of the Molonglo (Grillmair 2017) stream, and two newly detected streams that we name Elqui and Aliqa Uma. In addition, we find a long, diffuse structure that extends along the height of Q2 intersecting the Eridanus-Phoenix stellar overdensity lower in the DES footprint (Li et al. 2016). We name this structure the Palca stream and discuss it in more detail in Section 4.5.

\subsubsection{ATLAS Stream}

The ATLAS stream is a narrow stellar stream discovered in the first data release of the VST ATLAS survey, which covered a decl. slice around the stream from $-37^{\circ} \lesssim \delta_{2000} \lesssim-25^{\circ}$ to a limiting magnitude of $r \sim 22$ (Koposov et al. 2014). The ATLAS stream was later studied by Bernard et al. (2016) using the larger sky coverage provided by Pan-STARRS. The DES analysis is deeper than both VST ATLAS and Pan-STARRS, $g=23.5$, and extends the sky coverage around ATLAS to lower decl. The ATLAS stream does not appear to extend significantly beyond the length described by Koposov et al. (2014), ending at $\left(\alpha_{2000}, \delta_{2000}\right) \sim\left(30^{\circ} 7,-33^{\circ} .2\right)$. At higher decl. it becomes difficult to disentangle the ATLAS stream from the much more luminous Sagittarius stream before hitting the boundary of the DES footprint at $\left(\alpha_{2000}, \delta_{2000}\right) \sim\left(9.3,-20^{\circ} .9\right)$. Using Pan-STARRS data, Bernard et al. (2016) have extended ATLAS to $\delta_{2000} \sim-15^{\circ}$, leading to a total length of $\sim 28^{\circ}$, of which 22.6 is contained within DES.

We follow the procedure described in Section 3.2 to characterize the physical properties of the ATLAS stream. The deeper DES data prefer a slightly larger distance of

\footnotetext{
58 To honor the long astronomical tradition in Chile (home of the Blanco telescope), we name stellar streams in Q2 and Q3 after Chilean rivers and aquatic terms in native Chilean tongues. Aliqa Uma is the Aymara term for "peaceful water," and Willka Yaku is the Quechua term for "sacred water."
} 

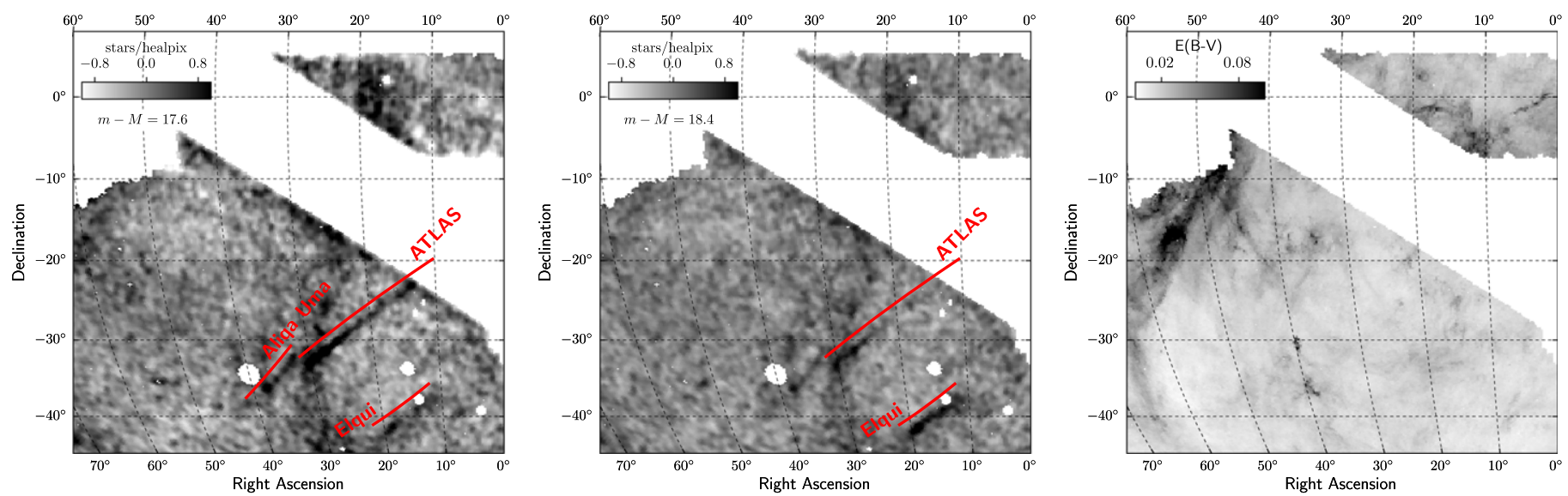

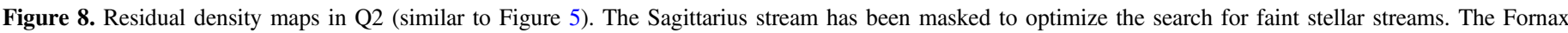

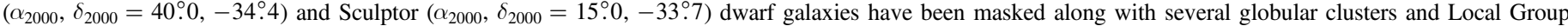

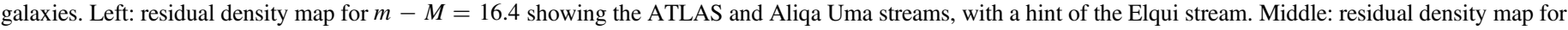

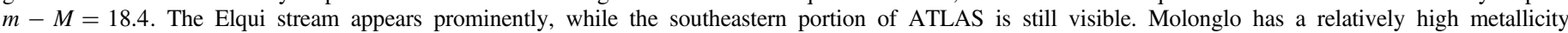

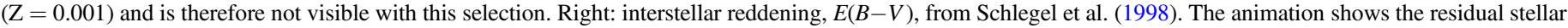
density map in Q2 as a function of distance modulus, from 14 to $19 \mathrm{mag}$.

(An animation of this figure is available.)

$22.9 \mathrm{kpc}$ ( $m-M \sim 16.8)$, which is marginally consistent with the previously measured distance, $20 \pm 2 \mathrm{kpc}$, derived using the VST ATLAS data (Koposov et al. 2014). At a distance of $22.9 \mathrm{kpc}$, the visible portion of the ATLAS stream extends to $9.0 \mathrm{kpc}$. We independently fit the distance modulus to each half of the ATLAS stream and find evidence that the southwestern portion of ATLAS has a distance modulus that is $\sim 0.3 \mathrm{mag}$ larger than the northeastern portion. The southwestern portion of ATLAS is detectable in the residual density maps at a distance modulus of $m-M>18.0$. The stellar density is not uniform along the length of ATLAS, and we note a roughly spherical overdensity in the southwestern portion at $\left(\alpha_{2000}, \delta_{2000}\right)=\left(25^{\circ} .37,-30^{\circ} .13\right)$. This overdensity is visible at lower significance in Figure 1 of Koposov et al. (2014). The DES data suggest a fainter absolute magnitude for the ATLAS stream, $M_{V}=-4.5$, compared to that estimated in the VST ATLAS data, $M_{V} \sim-6$ (Koposov et al. 2014).

Figure 10 shows the spatial distribution of stars in a coordinate system aligned with the endpoints of the ATLAS stream. Following the procedure described in Section 3.3, we fit the width of the ATLAS stream with a Gaussian model on top of a linear background. Our measured width of the ATLAS stream, $w=0.24$, is consistent with $w=0.25$ reported by Koposov et al. (2014), where $w$ corresponds to the Gaussian standard deviation. However, it is also clear in Figure 10 that the ATLAS stream deviates appreciably from a great circle on the sky, which would lie along the equator. We find that the ridgeline of the ATLAS stream is well described over the range $9.3<\alpha_{2000}<30^{\circ} .7$ by a second-order polynomial of the form

$$
\delta_{2000}=-15.637-0.545\left(\alpha_{2000}\right)-0.001\left(\alpha_{2000}\right)^{2} .
$$

Interestingly, Figure 10 also appears to show an underdensity in the stream at $\Lambda \sim 4^{\circ}$ that is approximately 2.5 in size. We caution that this underdensity occurs in a region where the polynomial background fit is complicated by the proximity of the Sagittarius stream. Oversubtraction of the background could manifest as an underdensity in the residual map. To check for the reality of this gap, we also analyzed the raw isochrone-selected counts without any background subtraction and found that in this region there is in fact a deficit with respect to the mean stream density. While the existence of this underdensity remains uncertain, if it is real, it could be due to perturbations by subhalos around the Milky Way (e.g., Ibata et al. 2002; Johnston et al. 2002). Erkal et al. (2016a) estimated the typical size and number of gaps in the ATLAS stream due to subhalos and found a characteristic gap size of $\sim 4^{\circ}$ with 0.1 gaps expected. However, this prediction depends on the length and orbital trajectory of the ATLAS stream; therefore, given the increased length of the ATLAS stream detected in this work and in Pan-STARRS (Bernard et al. 2016), as well as the uncertainty in its trajectory, the predicted number of gaps is likely an underestimate. If the underdensity is confirmed, then the gap can be used to infer the properties of the subhalo that created the gap (Erkal \& Belokurov 2015b), and the statistical properties of the stream density can be used to place constraints on the number of subhalos in the Milky Way (Bovy et al. 2017).

\subsubsection{Molonglo Stream}

The Molonglo stream was identified as a faint, narrow feature in data from Pan-STARRS (Grillmair 2017). We extrapolate Equation (2) from Grillmair (2017) to detect a narrow, $\sim 8^{\circ}$ extension of the Molonglo stream in the DES data offset by $\lesssim 2^{\circ}$. Molonglo is the only stream that is detected based on prior information from another survey, and there is some risk of confirmation bias. In fact, Molonglo is the least apparent feature in the residual density maps, due in part to its proximity to the Sagittarius stream. However, the Hess diagram for Molonglo shows a clear MS and MSTO (Figure 9), and the stream has a detection significance of 5.2 $\sigma$. After fixing the age ( $\tau=13.5$ Gyr $)$ and metallicity $(Z=0.0010)$ to match the Hess diagram, we fit the distance modulus and richness of Molonglo. We determine a distance of $22.9 \mathrm{kpc}(m-M=16.8)$, which agrees with the estimated distance of $20 \mathrm{kpc}$ from Grillmair (2017). However, the foreground subtraction in this region is difficult, and the remaining contamination may have artificially inflated the metallicity. Our best-fit width of 0.32 is narrower 

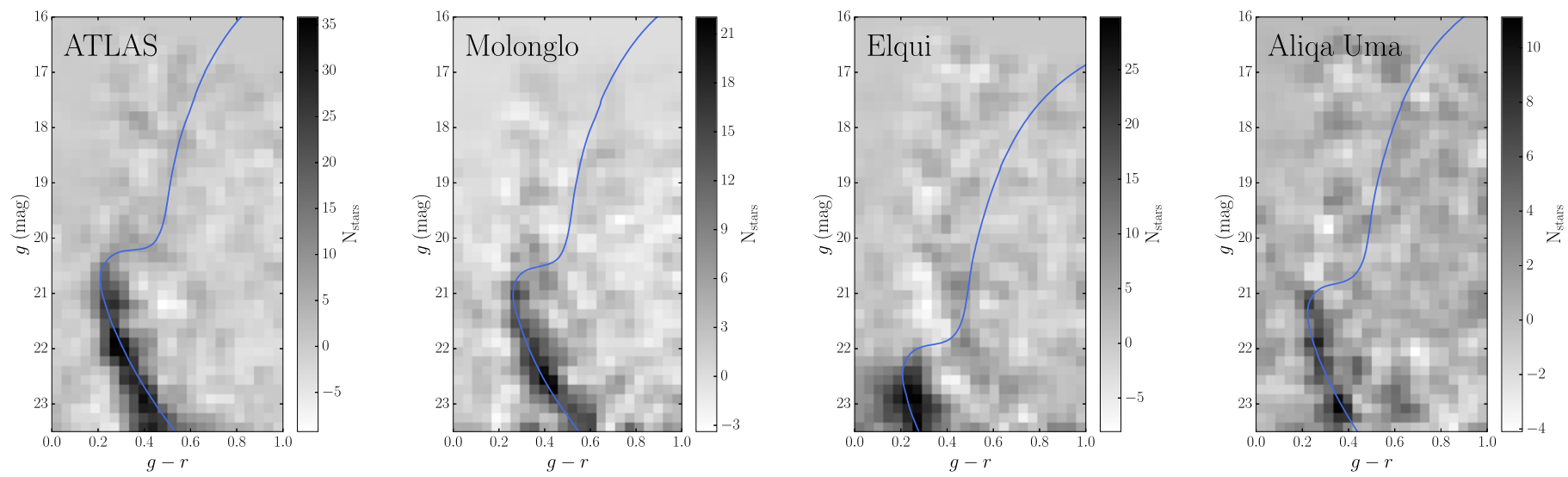

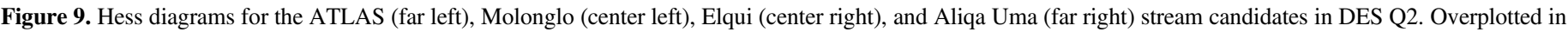
each panel is a Dotter et al. (2008) synthetic stellar isochrone with parameters determined from the best fit in Table 1 . These panels are similar to Figure 6.

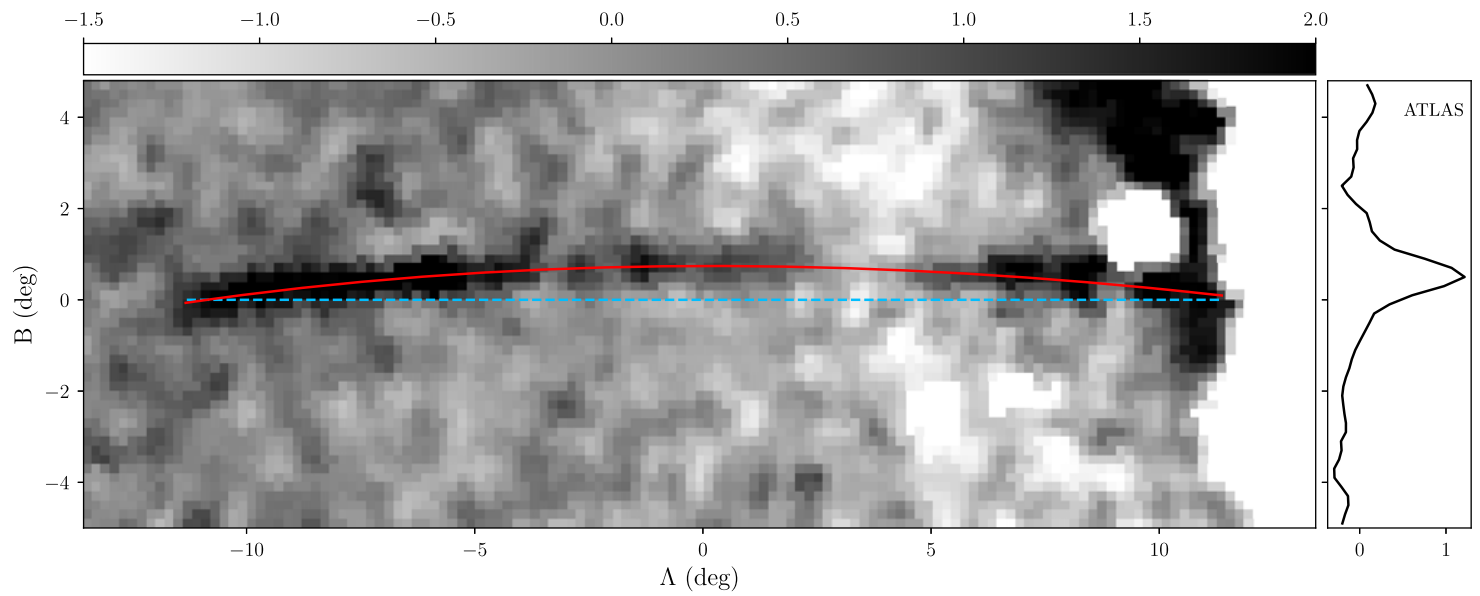

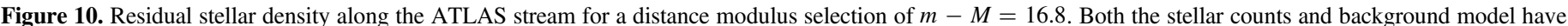

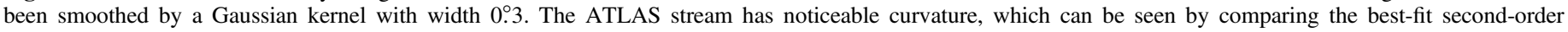
polynomial from Equation (6) (red solid line) to the plane of a great circle on the sky (blue dashed line).

than the $\sim 0.5$ reported by Grillmair (2017); however, it is unclear whether his width was measured after convolving with a 0.4 Gaussian kernel. We do not find evidence of the other three streams identified by Grillmair (2017).

\subsubsection{Elqui Stream}

At a heliocentric distance of $D_{\odot}=50.1 \mathrm{kpc}$, the Elqui stream is the most distant stream discovered in the DES Y3A2 data. Fitting the southwestern and northeastern halves of Elqui independently shows a shift in distance modulus from 18.2 to 18.5 , corresponding to a physical change in distance of $6.5 \mathrm{kpc}$ over a length of $8.2 \mathrm{kpc}$. The Elqui stream is broad and may show slight curvature on the sky, which is unexpected for a stream at large galactocentric radius. The background galaxy NGC 300 resides at $\left(\alpha_{2000}, \delta_{2000}\right) \sim\left(13.7,-37^{\circ} .7\right)$ but is unrelated to the much closer Elqui stream.

Elqui resides at similar galactocentric distance to the LMC and overlaps (in projection) the gaseous component of the Magellanic Stream (Nidever et al. 2008). Transforming to the Magellanic Stream coordinates defined by Nidever et al. (2008), the endpoints of Elqui are located at $L_{\mathrm{MS}}, B_{\mathrm{MS}} \approx$ $\left(-49^{\circ} .1,1^{\circ} .8\right),\left(-40^{\circ} .7,6^{\circ} .1\right)$. The proximity between Elqui and the Magellanic Stream suggests that Elqui may in fact be stellar ejecta from a past collision between the LMC and
SMC (Besla et al. 2010, 2012). Besla et al. (2012) suggest that a recent collision between the LMC and SMC could explain many of the observed features of the Magellanic system. If Elqui indeed formed as the result of such a collision, its existence could be used to further constrain the infall history of the Magellanic Clouds.

Evidence of stellar ejecta from the Magellanic Clouds is observed elsewhere in the DES footprint. The so-called "SMC northern overdensity" (Pieres et al. 2016) is located $\sim 8^{\circ}$ from the SMC on the southern edge of the DES footprint $\left(\alpha_{2000}, \delta_{2000}\right) \sim\left(15^{\circ},-65^{\circ}\right)$ and is visible in isochrone selections with $m-M \gtrsim 18$. Establishing complete DECam coverage around the Magellanic Clouds promises additional insight into how the Magellanic Clouds have shaped the Milky Way halo.

\subsubsection{Aliqa Uma Stream}

The Aliqa Uma stream resides at the southern end of the ATLAS stream, extending from $\alpha_{2000}, \delta_{2000} \sim\left(31^{\circ} .7,-31^{\circ} .5\right)$ to $\alpha_{2000}, \delta_{2000} \sim\left(40^{\circ} .6,-38^{\circ} .3\right)$. While the northern end of this stream is in close proximity to the southern end of the ATLAS stream, the difference in orientation and distance modulus, $m-M=17.3$, leads us to classify it as a distinct system rather than an extension of ATLAS. While this stream 

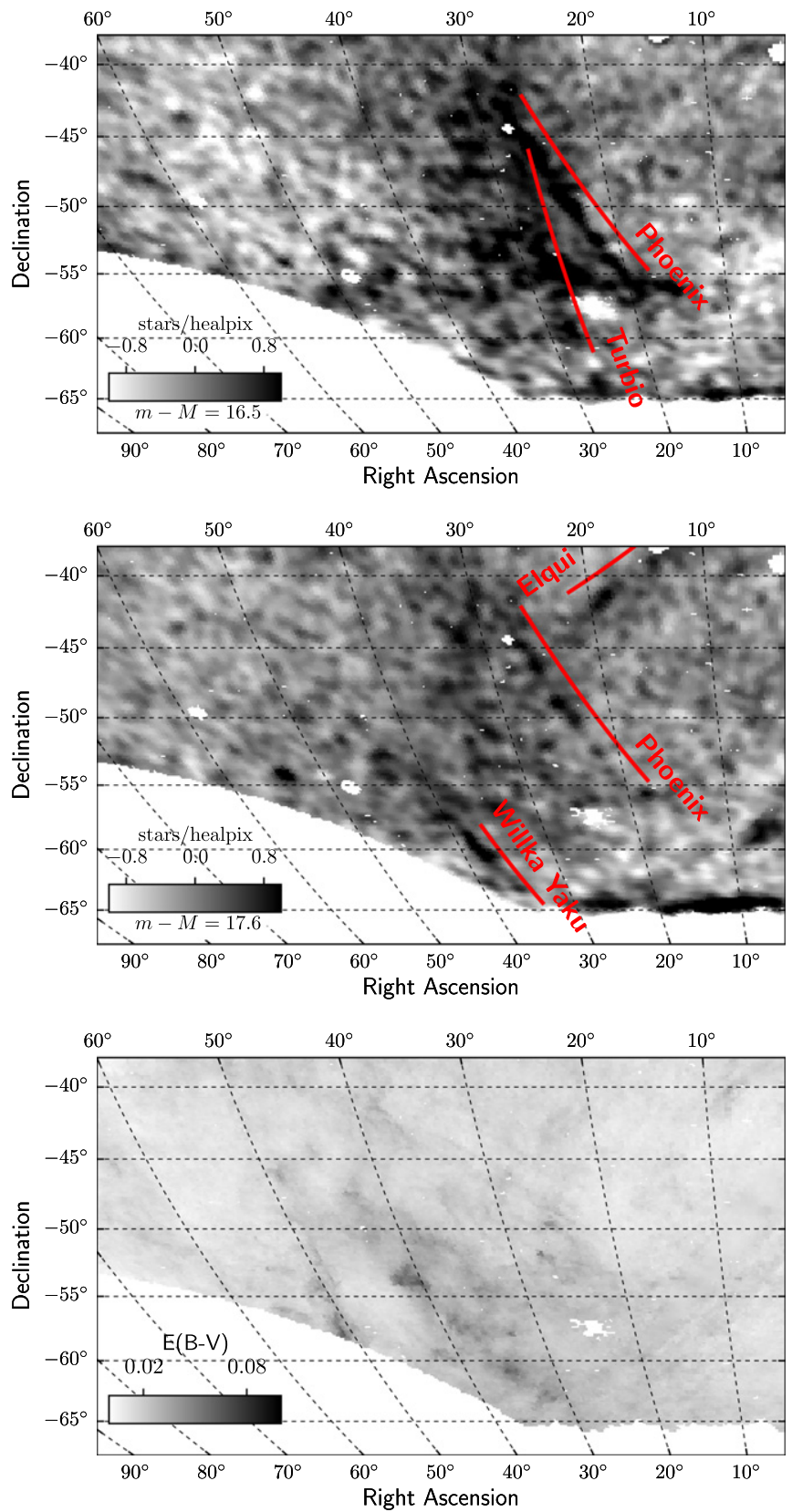

Figure 11. Residual density maps in Q3 (similar to Figure 5). Top: an isochrone selection with $m-M=16.5$ shows the Phoenix and Turbio streams superposed on the EriPhe overdensity. Middle: the Willka Yaku stream can be seen in a selection for $m-M=17.6$. Bottom: interstellar reddening, $E(B-V)$, from Schlegel et al. (1998). The animation shows the residual stellar density map in Q3 as a function of distance modulus, from 14 to 19 mag.

(An animation of this figure is available.)

crosses close to the Fornax dwarf galaxy in projection, it is substantially closer, and the two systems are very unlikely to be physically associated. The presence of Fornax and ATLAS bracketing the much fainter Aliqa Uma stream makes it difficult to establish a good background selection region.

\subsection{Third Quadrant}

The third DES quadrant (Q3) covers the region from $5^{\circ} \lesssim \alpha_{2000} \lesssim 60^{\circ}$ and $-65^{\circ} \lesssim \delta_{2000} \lesssim-42^{\circ}$ (Figure 11 ). This quadrant resides above the LMC and SMC and overlaps heavily with the EriPhe stellar overdensity (Li et al. 2016). Several linear structures are detected in this region; however, it is difficult to conclusively differentiate them from a diffuse component of EriPhe. In addition to the Phoenix stream (Balbinot et al. 2016), we identify two new stream candidates, Turbio and Willka Yaku.

\subsubsection{Phoenix Stream}

The Phoenix stream is a narrow stellar stream discovered in the DES Y1A1 data (Balbinot et al. 2016). Since the DES analysis of the Phoenix stream is discussed in great detail in Balbinot et al. (2016), we offer only a brief discussion here. The Y3A2 data provide a deeper and more complete catalog with improved photometric accuracy; however, the qualitative characteristics of the Phoenix stream are predominantly unchanged. It remains a clumpy and knotted stream, consisting of a more or less symmetric distribution of overdensities. The parameters that we derive for the Phoenix stream largely agree with those of Balbinot et al. (2016). We measure a distance of $19.1 \mathrm{kpc}(m-M=16.4)$, which is slightly larger than the value measured previously $(17.5 \pm 9 \mathrm{kpc})$. Similar to Balbinot et al. (2016), we find no indication of a distance gradient.

\subsubsection{Turbio Stream}

We find a linear feature near the center of the EriPhe stellar overdensity, which constitutes a candidate stream named Turbio. While Turbio is detected above the background of EriPhe with a significance of $7.9 \sigma$, it would be very surprising if the two were not physically associated. It is likely that Turbio stands out more prominently in our analysis compared to that of Li et al. (2016) owing to improved photometric calibration $(\sim 0.7 \%$ vs. $\sim 2 \%)$, increased depth $(g<23.5$ vs. $g<22.5$ ), and improved spatial resolution (smoothing kernel of 0.3 vs. 0.5$)$.

The MS of Turbio is detected in a Hess diagram that subtracts a neighboring region of EriPhe to the east of the structure (Figure 12). We fit an isochrone with fixed age and metallicity $(\tau=13.0 \mathrm{Gyr}, Z=0.0004)$ and find a best-fit distance modulus of $m-M=16.1$, which is indistinguishable from that of EriPhe and the nearby globular cluster NGC 1261. Li et al. (2016) suggest that EriPhe may have a common origin with the Virgo overdensity (Juric et al. 2008) and the HerculesAquila cloud (Belokurov et al. 2007a). However, the orientation of Turbio is nearly perpendicular with the orbit necessary to connect these three diffuse structures. In addition, Turbio may constitute a dense portion of the larger Palca structure that is seen to extend northward from the EriPhe cloud.

\subsubsection{Willka Yaku Stream}

Willka Yaku is a short and relatively narrow stream that extends $\sim 6^{\circ}$ from the southern edge of the DES footprint. Willka Yaku sits on the southeastern boundary of the EriPhe cloud, and we find a significant gradient in the stellar density transverse to the stream. To reduce the effects of foreground contamination, we fit only the more prominent northern half of the stream (Figure 12). A simultaneous fit of age, metallicity, and distance modulus yields a best-fit distance of $34.7 \mathrm{kpc}$ $(m-M=17.7)$. This places Willka Yaku at a significantly larger distance than EriPhe and makes a physical association less likely. 

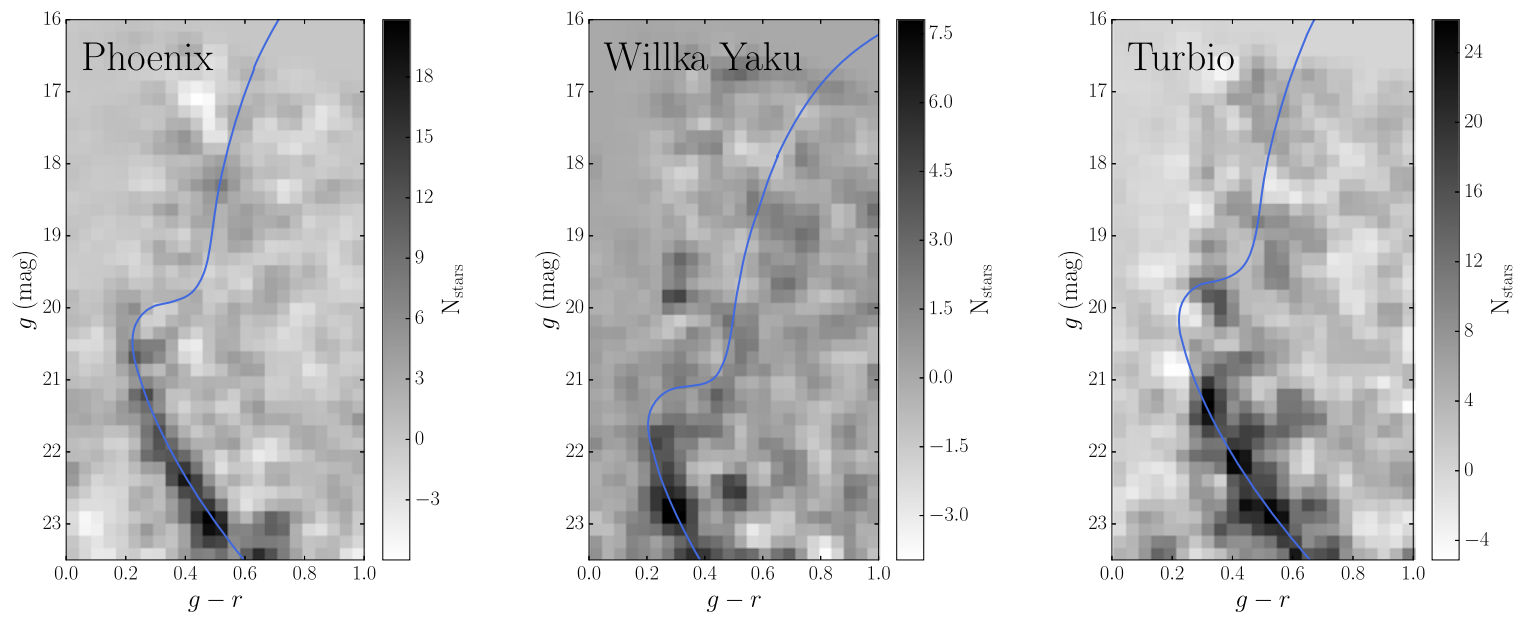

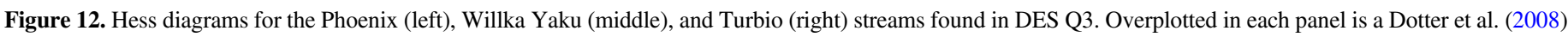
synthetic stellar isochrone with parameters determined from the best fit in Table 1 . These panels are similar to Figure 6.

\subsection{Fourth Quadrant ${ }^{59}$}

The fourth quadrant of DES (Q4) spans $60^{\circ} \lesssim \alpha_{2000} \lesssim 95^{\circ}$ and $-65^{\circ}<\delta_{2000}<-15^{\circ}$ (Figure 13). It contains the globular clusters NGC 1851 and NGC 1904, and structure from the Monoceros Ring can be seen in the direction of the Galactic anticenter. The proximity of the Monoceros and the Galactic plane makes background subtraction difficult near the eastern edge of this region. Inspection of the residual density maps yields two stellar stream candidates, Turranburra and Wambelong, which are reasonably wide $(w \sim 0.5)$ and detected at moderate significance.

\subsubsection{Turranburra Stream}

The Turranburra stream stretches across the northern portion of $\mathrm{Q} 4$, extending from $\left(\alpha_{2000}, \delta_{2000}\right)=\left(59^{\circ} .3,-18^{\circ} 0\right)$ to $\left(\alpha_{2000}, \delta_{2000}\right)=\left(75^{\circ} 2,-26^{\circ} .4\right)$. The Hess diagram of this stream shows a prominent MS and a hint of an RGB (Figure 14). We estimate that this structure resides at a distance of $27.5 \mathrm{kpc}$ and has a width of $288 \mathrm{pc}$. The physical width of Turranburra is more consistent with a dwarf galaxy progenitor, and the photometric estimate of metallicity of $Z=0.0003$ is comparable to photometric metallicities determined for ultrafaint galaxies (e.g., Bechtol et al. 2015). The extent of Turranburra is somewhat uncertain owing to its proximity to the edge of the DES footprint and its diffuse nature, especially at its northwestern end. The orbit of Turranburra appears to be distinct from other known streams and globular clusters, without any obvious association or potential progenitor.

\subsubsection{Wambelong Stream}

The Wambelong stream stretches northward from the eastern edge of the DES footprint, spanning $\sim 14^{\circ}$ from $\left(\alpha_{2000}\right.$, $\left.\delta_{2000}\right) \sim\left(90^{\circ} .5,-45^{\circ} .6\right)$ to $\left(\alpha_{2000}, \delta_{2000}\right) \sim\left(79^{\circ} .3,-34^{\circ} .3\right)$. The eastern extent of Wambelong is difficult to determine owing to confusion with foreground stars associated with the Galactic anticenter and the Monoceros Ring (Newberg et al. 2002; Yanny et al. 2003). The signature of Wambelong peaks at a heliocentric distance of $\sim 15.1 \mathrm{kpc}(m-M \sim 15.9)$,

\footnotetext{
59 To honor the long tradition of astronomy in Australia (stretching back tens of thousands of years), stellar stream candidates in this quadrant are named after aboriginal terms for rivers in Australia.
}

with no strong indication of a distance gradient. A residual overdensity at $\left(\alpha_{2000}, \delta_{2000}\right) \sim\left(70^{\circ} .4,-23^{\circ} .9\right)$ is aligned with the Wambelong stream and may suggest that this stream is nearly twice as long as our conservative estimate.

\subsection{Diffuse Overdensities}

While the search described here is optimized for $\lesssim 1^{\circ}$-wide stellar features, we note that we are sensitive to more diffuse stellar systems. Without the directionality of a narrow stream, interpreting the origin and physical parameters of these diffuse structures becomes much more difficult. However, we do note that the DES Y3A2 data unambiguously confirm the existence of the EriPhe overdensity ( $\mathrm{Li}$ et al. 2016) and show strong indications of additional substructure around or within this system. Apart from the aforementioned Phoenix, Turbio, and Willka Yaku streams, there are several lower-significance linear structures that overlap EriPhe at a heliocentric distance of $\sim 16.6 \mathrm{kpc}(m-M=16.1)$. One pronounced feature runs nearly east-west with $\delta \approx-56^{\circ}$ (Figure 11). Unfortunately, image-level masking around the super-saturated star Achernar $\left(\alpha_{2000}, \delta_{2000}=24^{\circ} .43,-57^{\circ} .24\right)$ complicates the interpretation of the southern portion of EriPhe.

The Palca ${ }^{60}$ stream is a broad curvilinear overdensity in the Y3A2 data that extends northward from EriPhe at $\alpha_{2000} \sim 30^{\circ}$. Palca extends from the Turbio stream in the south to the northern boundary of the DES footprint, crossing the Sagittarius stream at $\alpha_{2000} \sim 20^{\circ}$. Palca is more diffuse than the other streams discovered in DES Y3A2 (FWHM $\sim 2^{\circ}$ ). To increase our sensitivity to this broad feature, we convolve the residual density maps by a $1^{\circ}$ kernel and plot in equatorial coordinates (Figure 15). We find that the northern part of Palca is at a significantly larger distance, $\sim 36 \mathrm{kpc}(m-M \sim 17.8$ ), than EriPhe. It is unclear whether this is a signature of a distance gradient from EriPhe to Palca, or whether these are two distinct systems. Assuming that Palca spans the DES footprint (i.e., overlapping with EriPhe), the ridgeline of this structure can be approximated with a second-order polynomial of the form

$$
\alpha_{2000}=17.277-0.495 \delta_{2000}-0.0046 \delta_{2000}^{2} .
$$

\footnotetext{
${ }^{60}$ Palca is the Quechua word for "cross of rivers."
} 

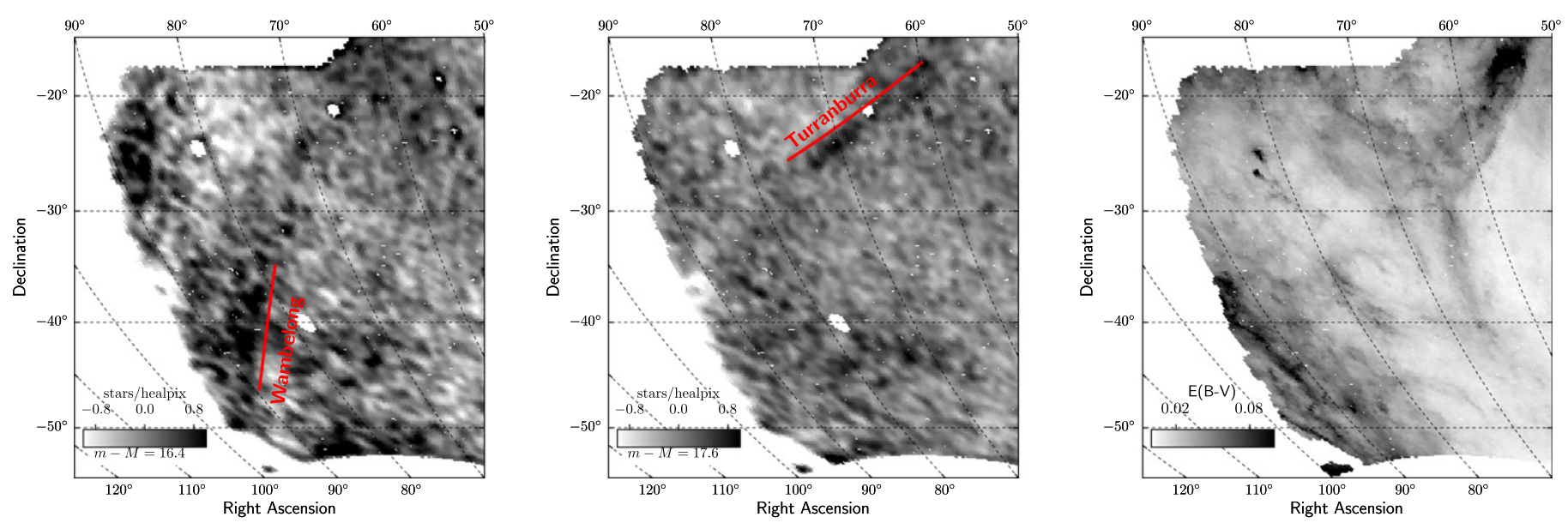

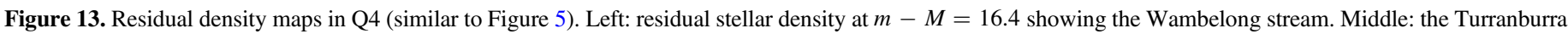

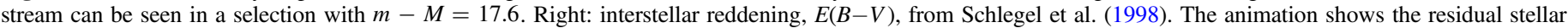
density map in Q4 as a function of distance modulus, from 14 to 19 mag.

(An animation of this figure is available.)
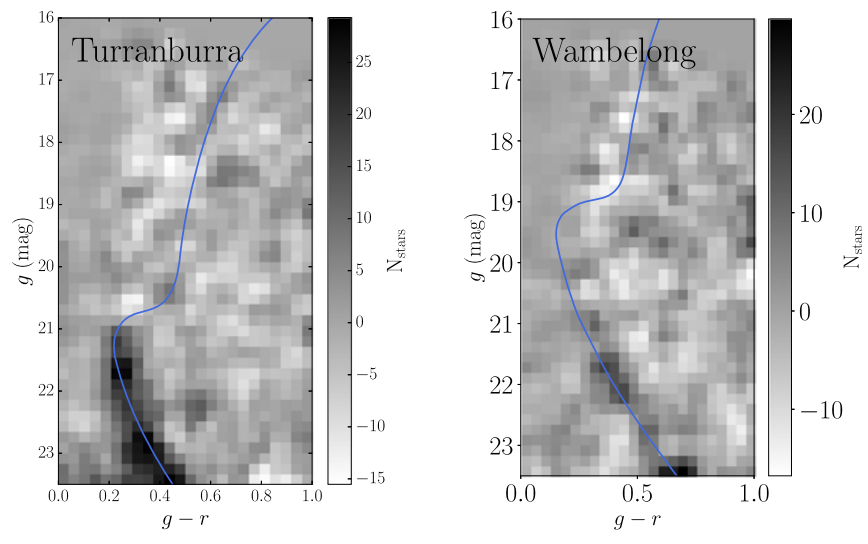

Figure 14. Hess diagrams for the Turranburra (left) and Wambelong (right) stream candidates found in DES Q4. Overplotted in each panel is a Dotter et al. (2008) synthetic stellar isochrone with parameters determined from the best fit in Table 1. These panels are similar to Figure 6.

The above equation is found to be valid for $-55^{\circ}<$ $\delta_{2000}<2^{\circ}$.5. Palca shows appreciable curvature on the sky, suggesting that it may be in a modestly elliptical orbit. The orbital trajectory of Palca is strongly misaligned with the proposed polar orbit connecting EriPhe with the Virgo overdensity and the Hercules-Aquila cloud (Li et al. 2016). A physical association between Palca and EriPhe would disfavor this hypothesis. However, the orbit of Palca may be broadly consistent with another scenario proposed by Li et al. (2016), suggesting that EriPhe may be the remnants of a disrupted dwarf galaxy originally associated with NGC 1261 and the Phoenix stream.

\subsection{Globular Clusters}

The DES Y3A2 footprint contains five classical globular clusters (NGC 288, NGC 1261, NGC 1851, NGC 1904, and NGC 7089) and four more distant clusters (Whiting 1, AM-1, Eridanus, and Reticulum). While a full investigation of globular clusters is outside the scope of the current paper, we note that our analysis is sensitive to stellar features around these clusters. Four of the classical globular classical clusters
(NGC 288, NGC 1261, NGC 1851, and NGC 1904) show hints of extended stellar structure. ${ }^{61}$ These features are detectable with the generic isochrone selection described in Section 3 and can be seen in the animations associated with Figure 4. However, to optimize our sensitivity to faint features, we built individual matched-filter selections for each cluster using the CMD of stars within an annulus of 4 ! $2<r<7$ !.2 around each cluster. We create an optimal weighting by taking the ratio between the density (in color-magnitude space) of cluster member stars compared to the Milky Way foreground population averaged over the DES footprint,

$$
w_{i, j}=f_{\mathrm{gc}}(i, j) / f_{\mathrm{mw}}(i, j),
$$

where $i, j$ index the color and magnitude bins, $f_{\mathrm{gc}}(i, j)$ is the normalized density of cluster stars per bin, $f_{\mathrm{mw}}(i, j)$ is the normalized density of Milky Way stars, and $w_{i, j}$ is the weighting (Rockosi et al. 2002). We mask circular regions comparable to the Jacobi radii of each cluster (Table 4) and convolve the selected stellar density with a Gaussian kernel with $\sigma=0^{\circ} .25$. We follow the same procedure to derive a global polynomial fit to the smoothed density of selected stars and create residual density maps from the difference between the data and the polynomial fit (Figure 16).

We compare the observed stellar features to predictions about orbital motion and tidal tail formation in each globular cluster. We simulated the orbits of the globular clusters using the spray-particle implementation by Küpper et al. (2012), where the escape velocity was modified to match that observed in $N$-body simulations with realistic tidal fields (Claydon et al. 2017). We assume a Milky Way potential similar to the best-fit Palomar 5 model (Küpper et al. 2015), but with a Jaffe bulge. The cluster initial mass is obtained using the method outlined in Balbinot \& Gieles (2018). The tidal tail formation was simulated for the last $6 \mathrm{Gyr}$ of the cluster history, and particles were released every $1 \mathrm{Myr}$.

\footnotetext{
${ }^{61}$ NGC 7089 (M2) is located in the narrow Stripe 82 region of the DES footprint. The narrow width of this region and the large density of foreground stars make a search for extended structure challenging.
} 


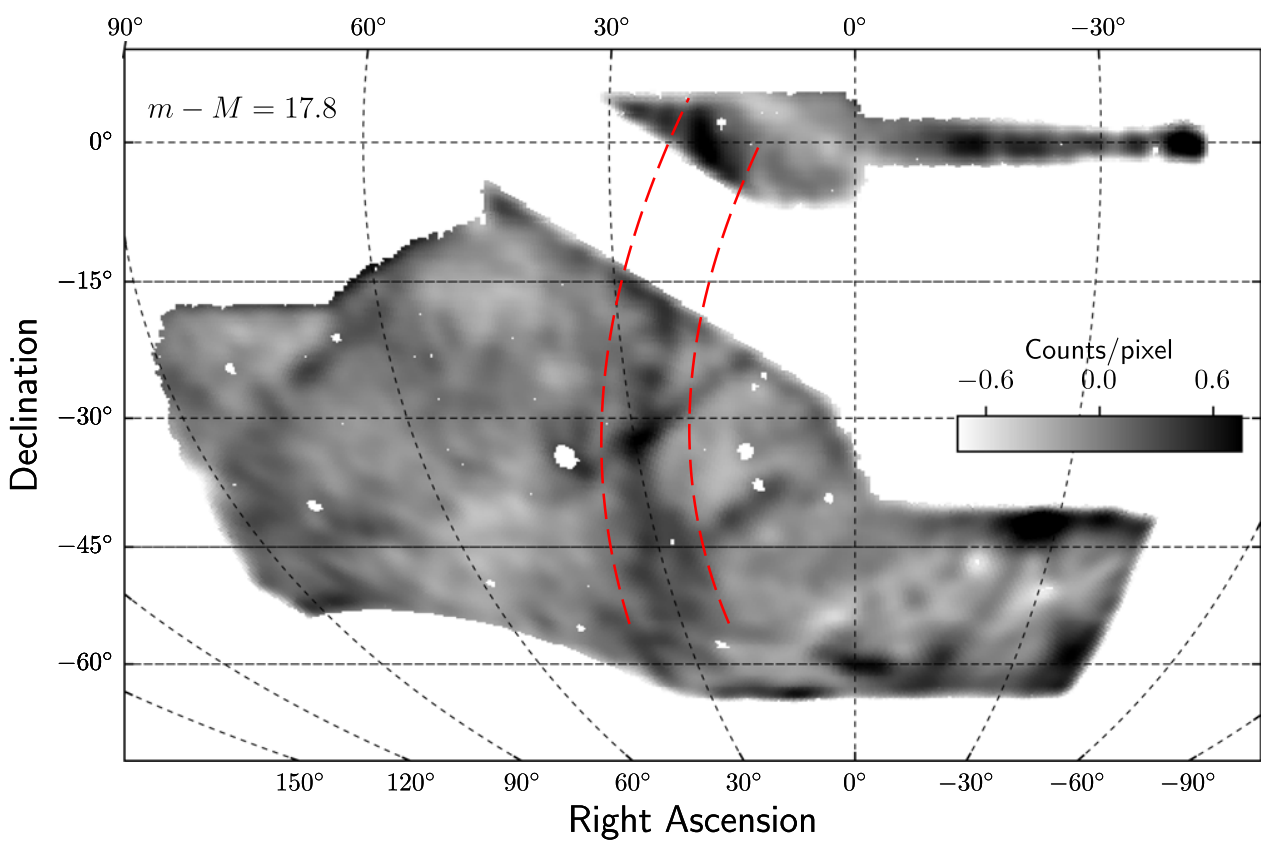

Figure 15. Residual density map for an isochrone selection at $m-M=17.8$ smoothed by a $1^{\circ}$ Gaussian kernel. The Palca overdensity (bracketed in red) runs along the north-south direction at $\alpha_{2000} \sim 30^{\circ}$. At low decl. Palca overlaps the EriPhe stellar overdensity with roughly the same orientation as the Turbio stream. The ATLAS, Elqui, Tucana III, Chenab, Willka Yaku, and Turranburra streams are also visible in this map.

The heliocentric distance, sky position, line-of-sight velocity, and integrated magnitude for each cluster were taken from Harris (1996, updated 2010). Proper motions for NGC 288 and NGC 1851 are taken from Dinescu et al. (1997), for NGC 1904 we used values from Dinescu et al. (1999), and for NGC 1261 we used values from Dambis (2006). These parameters are summarized in Table 4. Our simulations assumed a galactocentric solar position of $(8.3 \mathrm{kpc}, 0,0)$, a local reflex motion of $U, V, W=(11.1,12.24,7.25) \mathrm{km} \mathrm{s}^{-1}$ (Schönrich et al. 2010), and a circular solar velocity of $U, V, W=(0,233,0) \mathrm{km} \mathrm{s}^{-1}$ (Küpper et al. 2015).

\subsection{1. $N G C 288$}

NGC 288 is a globular cluster with a dynamical evolution that is strongly driven by tidal shocks (Gnedin \& Ostriker 1997). Grillmair et al. (1995) showed initial evidence for extratidal features using photographic photometry. The analysis of NGC 288 was extended to a larger field by Leon et al. (2000), who reported evidence of two sets of tidal tails, extended along the direction of motion and in the direction of the Galactic center. Subsequently, Grillmair et al. (2004) used Two Micron All Sky Survey data to suggest a $\sim 17^{\circ}$ tidal tail; however, this claim was later refuted by Piatti (2018) using deeper data from Pan-STARRS PS1. In contrast, Piatti (2018) found evidence for clumpy extratidal structure extending $120 \mathrm{pc}(0.8)$ from the cluster center. The DES Y3A2 data are deeper than the Pan-STARRS data analyzed by Piatti (2018) and support previous reports of clumpy extratidal structure extending $\sim 1.5$ from the core of NGC 288 . In addition, there is evidence that these extratidal features may extend $\sim 5^{\circ} .5$ southward of NGC 288 (Figure 16). This structure is misaligned with the orbital motion of NGC 288 and the vector connecting NGC 288 to the Galactic center.
4.6.2. $N G C 1261$

NGC 1261 resides in the southern portion of the DES footprint, and it has been suggested that it may be associated with the Phoenix stream (Balbinot et al. 2016) and/or the EriPhe stellar overdensity (Li et al. 2016). The analysis of Leon et al. (2000) suggests the existence of a tidal tail oriented in the direction of the Galactic center. Recent observations with DECam have similarly detected evidence that the stellar halo of NGC 1261 extends beyond its nominal Wilson tidal radius (Carballo-Bello et al. 2018; Kuzma et al. 2018) but do not see any evidence of tail-like structure. Our observations support the existence of extratidal structure around NGC 1261. While some of this structure appears to be aligned with the orbital motion of the cluster, it is difficult to draw any firm conclusion without more detailed analysis.

\subsubsection{NGC 1851}

Tidal tails were reported around NGC 1851 by Leon et al. (2000), who claimed to detect low surface brightness features oriented with the direction of motion of the cluster. However, Olszewski et al. (2009) found no evidence of tidal tails in a more recent analysis of deeper data. Rather, Olszewski et al. (2009) reported a low surface brightness extended stellar halo extending to $\sim 1^{\circ} 25$. Our residual density maps show evidence of both an extended stellar halo around NGC 1851 and a set of faint linear features aligned with the predicted orbit (Figure 16). The residual stellar density extends prominently to a radius $>1^{\circ}$ from the cluster core, agreeing with measurements of an extended stellar halo by Olszewski et al. (2009). The linear feature extends at least $5^{\circ}$ to the north and south of NGC 1851. The orientation of these features is well aligned with the orbital motion of NGC 1851, suggesting that these features may be tidal tails. In fact, the animation associated with Figure 4 suggests that these putative tidal tails may extend $\pm 15^{\circ}$ or more 
Table 4

Globular Cluster Parameters

\begin{tabular}{|c|c|c|c|c|c|c|}
\hline Name & $\begin{array}{l}\alpha_{2000} \\
(\mathrm{deg})\end{array}$ & $\begin{array}{l}\delta_{2000} \\
(\mathrm{deg})\end{array}$ & $\begin{array}{c}\mathrm{D}_{\odot} \\
(\mathrm{kpc})\end{array}$ & $\begin{array}{c}r_{\mathrm{J}} \\
(\mathrm{pc})\end{array}$ & $\begin{array}{c}\mu_{\alpha} \cos (\delta) \\
\left(\operatorname{mas~yr}^{-1}\right)\end{array}$ & $\begin{array}{c}\mu_{\delta} \\
\text { (mas yr }^{-1}\end{array}$ \\
\hline NGC 288 & 13.189 & -26.583 & 76.4 & 8.9 & 4.48 & -6.04 \\
\hline NGC 1261 & 48.068 & -55.216 & 146.4 & 16.3 & 1.33 & -3.06 \\
\hline NGC 1904 & 81.046 & -24.525 & 153.8 & 8.9 & 2.12 & -0.02 \\
\hline
\end{tabular}

Note. Centroids, heliocentric distances, Jacobi radii, and proper motions for four classical globular clusters in the DES footprint. Values are taken from Harris (1996, 2010 edition), Balbinot \& Gieles (2018), Dinescu et al. (1997), Dambis (2006), and Dinescu et al. (1999).

from NGC 1851. The detection of extratidal structure associated with NGC 1851 agrees with recent work by Kuzma et al. (2018) and Carballo-Bello et al. (2018). The DES data greatly extend the coverage around NGC 1851 and make a strong case for a "vast stellar structure" (Carballo-Bello et al. 2018) extending both northward and southward of this cluster.

\subsection{4. $N G C 1904$}

An extended halo of extratidal stars around NGC 1904 was first recognized by Grillmair et al. (1995) and later by Leon et al. (2000). We confirm the existence of extratidal structure extending $\sim 1.5$ from the cluster center. Leon et al. (2000) suggest that the short relaxation time of NGC 1904 would cause mass segregation in the tidal tails. This may explain why these features were not seen with shallower observations. Interestingly, these structures appear to be symmetric but are misaligned with the orbital motion of the cluster. Observations of NGC 1904 by Carballo-Bello et al. (2018) reach a similar conclusion that the stellar distribution of this cluster deviates from the conventional King and Wilson models to fill, and slightly overflow, the Jacobi radius of the cluster.

\section{Discussion}

\subsection{Potential Associations with Known Systems}

We use the recent catalog of stellar streams compiled by Mateu et al. (2018), augmented with the recently discovered Jet stream (Jethwa et al. 2017), to assess whether any of our stream candidates may be associated with previously detected streams located in other regions of the sky. We begin by transforming the endpoints of each stream into galactocentric Cartesian coordinates. The ellipticity of these streams is poorly constrained, so we assume only that the streams orbit in a plane around the Galactic center. We find the pole of each stream, $(\phi, \psi)$, defined as the positive normal vector of a plane containing both endpoints. Uncertainties in the pole location for our newly found streams were estimated by assuming a $20 \%$ uncertainty on the heliocentric distance before converting to galactocentric coordinates. The poles for the new and previously discovered streams are shown in Figure 17.

The distribution of DES stream poles shown in the left panel of Figure 17 is clearly nonuniform. We do not find any strong association of stream poles coinciding with the proposed vast polar structure (VPOS; Pawlowski et al. 2012, 2015). Specifically, in the left panel of Figure 17 we plot the VPOS +new pole (Table 1 of Pawlowski et al. 2015) transformed into galactocentric coordinates assuming a distance of $100 \mathrm{kpc}$. However, the limited sky coverage of the DES footprint will bias the observable distribution of stream poles. To estimate this bias, we generate a uniform random sample of galactocenric great-circle orbits with a radius of $25 \mathrm{kpc}$. We calculate the fraction of each great-circle orbit contained within the DES footprint as a function of galactocentric orbital pole and show this in the right panel of Figure 17. We find that the observed distribution of stream poles is consistent with the predictions from our simple simulation. The DES footprint is clearly biased against detecting streams having poles with $\psi_{\text {pole }}<30^{\circ}$ and $-60^{\circ}<\phi_{\text {pole }}<120^{\circ}$. We find qualitatively similar results for random samples of orbits with galactocentric radii of 15 and $50 \mathrm{kpc}$.

To investigate potential associations for the new DES streams, we plot galactocentric great-circle orbits for the DES streams and other known streams with similar orbital poles (Figure 18). Full phase-space information is necessary to definitively match between streams systems; however, we do note several tentative associations based on the photometrically measured properties of the DES streams. Figure 18 shows a strong correspondence between Ravi and the tentative candidate RR Lyrae stream 24.5-1 (Mateu et al. 2018). The poles of these two stream candidates match within $3^{\circ}$, while their distance moduli differ by $\Delta(m-M) \sim 0.2$ mag (well within the systematic error associated with our isochrone fitting). Such an association supports the robustness of tentative candidates identified below the conservative $>4 \sigma$ significance threshold of Mateu et al. (2018).

The orbital pole of the Hermus stream (Grillmair 2014) is only 3.4 from that of Willka Yaku, but the galactocentric distances of the two streams differ by $\sim 16 \mathrm{kpc}$. It has previously been suggested that Hermus may be a northern extension of the Phoenix stream (Grillmair \& Carlberg 2016), which resides along a slightly different orbital plane but is well matched in distance. Kinematic information would help to resolve this ambiguity.

Globular clusters and dwarf galaxies may provide possible progenitors for the newly discovered streams. The globular cluster candidate with the smallest separation is IC 4499. It is within 1.2 of the great-circle orbit of Turbio and has a galactocentric radius that differs by less than $2 \mathrm{kpc}$. IC 4499 is a moderate-mass, low-density cluster with signatures of an extratidal stellar halo (Walker et al. 2011). Cetus II, an ultrafaint dwarf galaxy (Drlica-Wagner et al. 2015), is another candidate for association. It lies 1.7 off of the great-circle orbit of Aliqa Uma, has a galactocentric radius that is $1.1 \mathrm{kpc}$ larger, and is located $43^{\circ}$ from the nearest endpoint of the stream. We note also that the tidal tails of Palomar $5\left(R_{\mathrm{GC}}=18.2 \mathrm{kpc}\right)$ have an orbital pole within $6^{\circ}$ of the ATLAS stream $(25 \mathrm{kpc})$. 
NGC 288

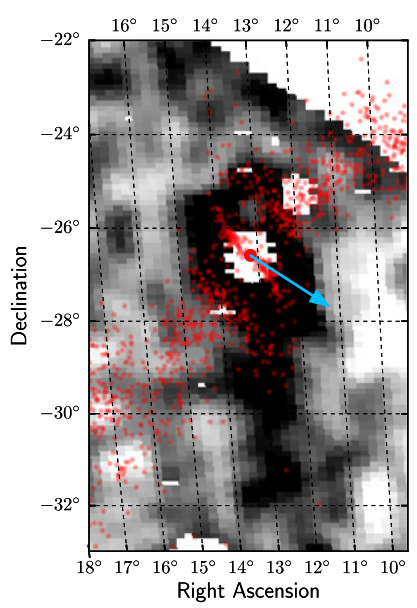

NGC 1261

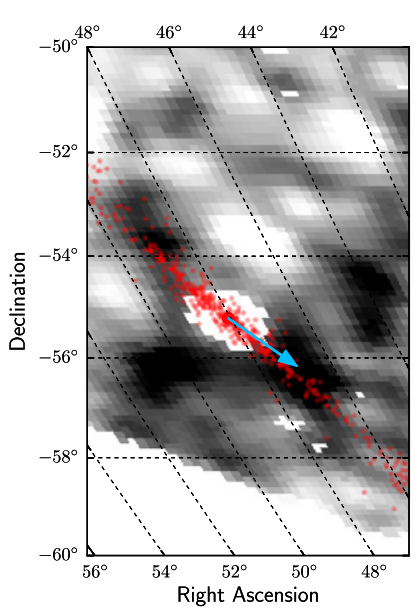

NGC 1851

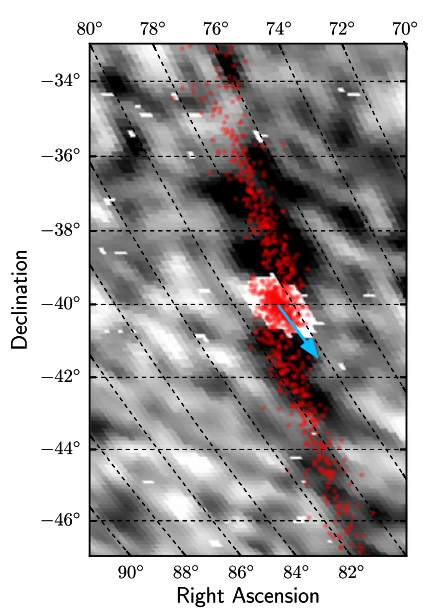

NGC 1904

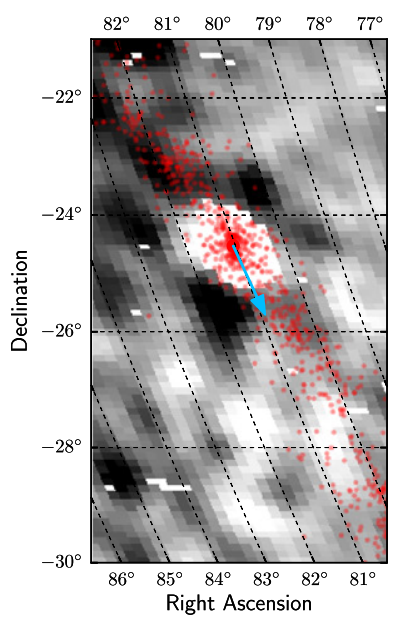

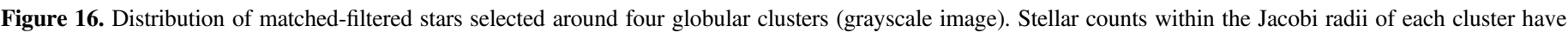

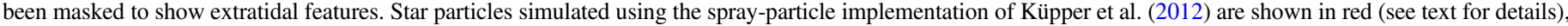

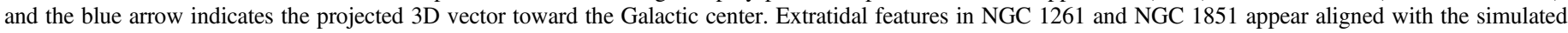
orbit; however, this is not the case for the other two systems.

Agnello (2017) identified four stellar stream candidates overlapping the DES footprint using a WISE-Gaia multiple search. It was proposed that two of these streams, WG3 and WG4, may have associated counterparts in DES (Agnello 2017). While WG3 and WG4 appear qualitatively similar to Indus and Jhelum, their absolute coordinates are offset by $\Delta \alpha_{2000} \sim-20^{\circ}$ (an angular separation of $\sim 15^{\circ}$ ). We do not see any significant stellar overdensities associated with the positions of WG3 or WG4 reported by Agnello (2017), though their proximity to the Galactic plane and unknown distance make it difficult to quantify the lack of a DES counterpart. If we allow $\Delta \alpha_{2000}$ offsets of $\sim 10^{\circ}$, then we find a possible correspondence between WG1 and Wambelong. WG1 is offset from Wambelong by $\Delta \alpha_{2000} \sim-8^{\circ}$ (angular separation of $\sim 6^{\circ}$ ) and extends both northeast and southwest along a similar path. This observation provides circumstantial evidence in support of the longer extent of Wambelong proposed in Section 4.4.2. WG2 does not correspond to any of the highsignificance stream candidates reported here. However, WG2 appears qualitatively similar to a lower-significance feature found to the southwest of NGC 1851 extending from $\alpha_{2000}, \delta_{2000}=(56.1,-50.4)$ to $(78.5,-40.0)$ at a distance modulus of $m-M \sim 17.5$ (animation of Figure 4). We expect Gaia DR2 to greatly improve the power of stellar stream searches using astrometric techniques.

\subsection{Milky Way Gravitational Potential}

Stellar streams can be used to constrain the Milky Way gravitational potential (e.g., Johnston et al. 2005; Koposov et al. 2010; Law \& Majewski 2010; Gibbons et al. 2014; Bowden et al. 2015; Küpper et al. 2015; Bovy et al. 2016). Full potential modeling is beyond the scope of this work; however, we note that the streams discovered by DES span a wide range of galactocentric radii and should be able to constrain how the Milky Way's density profile and shape evolve with radius. In this context, we expect that the ATLAS stream will be especially useful since it is long and does not lie on a great circle (see Figure 10).

However, even without sophisticated modeling we can make some general observations in the context of the Milky Way potential. Erkal et al. (2016b) suggest that the connection between stream width and orbital inclination could provide an independent constraint on the symmetry axis and flattening of the Milky Way halo. In Figure 19, we plot the angular stream width (as would be observed from the Galactic center) against the galactocentric polar angle, $\psi$, and the galactocentric azimuthal angle, $\phi$. This figure shows a large scatter, which is to be expected from a heterogeneous population of progenitors. While it is interesting to note that the streams that appear the widest are also on nearly polar orbits, interpreting any trend in this figure is subject to a number of caveats.

In particular, inferences involving stream widths rely on the assumed mass, structural properties, orbit, and dynamical age of the stream and its progenitor. Geometric effects can cause debris in the stream plane to contribute to the perceived width as calculated by a heliocentric observer. Furthermore, the width can fluctuate along a stream owing to the existence of nodes between the progenitor plane and the planes of the stream debris. Nonetheless, it is interesting to note that Figure 19 is in general agreement with a model where the Galactic symmetry axis is perpendicular to the plane of the disk. ${ }^{62}$

\subsection{Stream Progenitors}

In Table 2 we provided estimates of the stellar mass and progenitor mass (based on the stream width; see Erkal et al. 2016b) of each stream. These can be combined to give a mass-to-light ratio for the progenitor. Doing this, we find that five of the streams (Tucana III, ATLAS, Phoenix, Willka Yaku, and Turbio) have mass-to-light ratios less than $\sim 30$, while the other eight streams have significantly higher mass-to-light ratios. Given this seeming dichotomy, it is possible that the progenitors with low mass-to-light ratios are globular clusters while those with the higher ratios are dwarf galaxies. However, since the mass-to-light ratios are all higher than expected for a globular cluster (Phoenix stream has the lowest ratio of 10), we cannot make any firm conclusions.

\footnotetext{
$\overline{62}$ We note that Figure 16 in Erkal et al. (2016b) uses an inconsistent convention for the sign of the azimuthal coordinate $\phi$.
} 


\begin{tabular}{ccccc}
\multicolumn{5}{c}{ Orbit Containment Fraction } \\
\hline 0.0 & 0.1 & 0.2 & 0.3 & 0.4
\end{tabular}
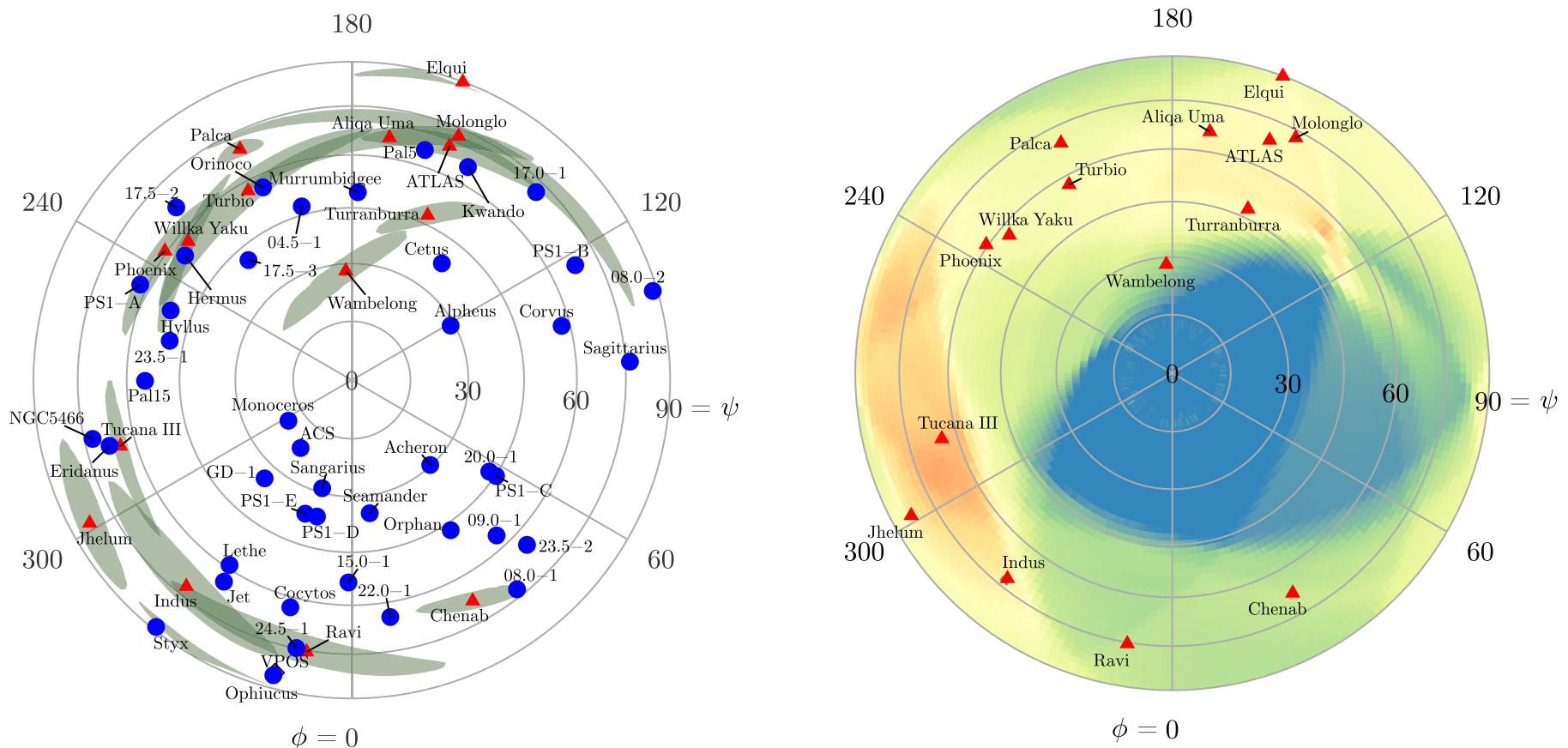

Figure 17. Left: galactocentric orbital poles for DES stream candidates (red triangles) and previously known streams (blue circles; Jethwa et al. 2017; Mateu et al. 2018). Green shaded regions represent the $1 \sigma$ uncertainty ellipses for the poles of the DES streams assuming a distance uncertainty of $20 \%$. The purple diamond shows the mean orbital pole of the proposed vast polar structure (Pawlowski et al. 2015). Right: fraction of uniformly distributed galactocentric great-circle orbits with $R_{\mathrm{GC}}=25 \mathrm{kpc}$ that are contained within the DES footprint. The DES footprint imposes a geometric bias against the detection of streams with $-60^{\circ}<\phi_{\text {pole }}<120^{\circ}$ and $\psi_{\text {pole }} \lesssim 30^{\circ}$.

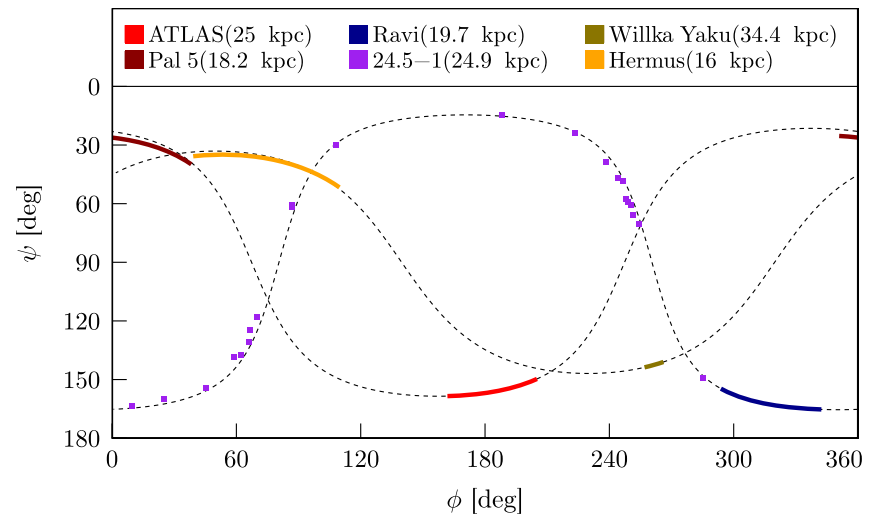

Figure 18. Great-circle orbital models for three of the closest associations between DES and previously detected streams are plotted in galactocentric coordinates $(\phi, \psi)$. The dotted lines are great-circle orbits derived from the DES stream endpoints. The solid colored lines show the locations of the streams themselves; the purple squares show the RR Lyrae (Mateu et al. 2018) that make up the stream 24.5-1.

These higher-than-expected mass-to-light ratios could be due to a variety of reasons. As discussed in 3.3, the progenitor mass estimate is only approximate and only works on average for streams on eccentric orbits. Furthermore, if the stream widths have fanned out owing to evolving in a nonspherical potential (e.g., Erkal et al. 2016b), the inferred progenitor masses will be overestimated. Finally, the stellar masses will be underestimated for streams that are not fully contained within the DES footprint.

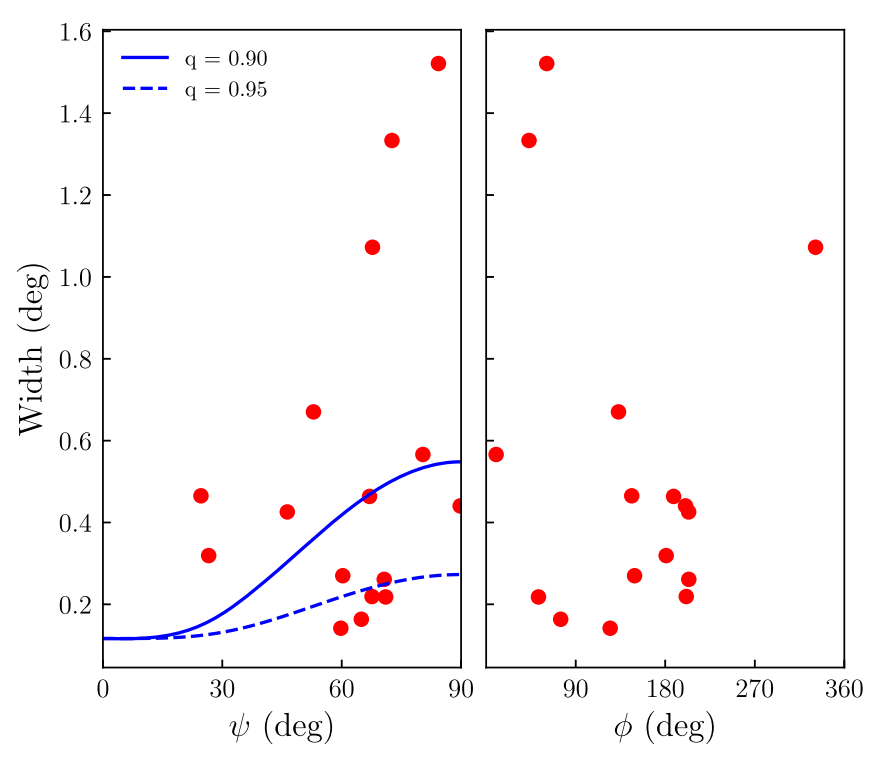

Figure 19. Angular widths of stellar streams detected by DES (as perceived by an observer at the Galactic center) as a function of their galactocentric pole orientation. The left and right panels show the stream width as a function of the galactocentric polar and azimuthal angle, respectively. The curves show the expected stream width from Erkal et al. (2016b) for streams with age 4 Gyr in a potential with two different flattenings, $q=0.9$ or $q=0.95$. These curves are produced assuming a progenitor with a mass of $10^{4.5} M_{\odot}$ on an orbit with a pericenter of $15 \mathrm{kpc}$ and an apocenter of $30 \mathrm{kpc}$ in a logarithmic potential with a circular velocity of $220 \mathrm{~km} \mathrm{~s}^{-1}$. The bunching of the polar angles of observed streams around low azimuthal angles is caused by the coverage of DES. Interestingly, the widest streams (Jhelum, Indus, Ravi, and Chenab) are close to polar orientations. 


\section{Conclusions}

We searched for Milky Way stellar streams by applying a matched filter for old, metal-poor stellar populations to $3 \mathrm{yr}$ of data from DES. The unprecedented photometric calibration, depth, and coverage area of the DES data allow us to detect stellar streams out to a distance of $>50 \mathrm{kpc}$. Our analysis recovers four narrow stellar streams previously identified within the DES footprint. In addition, we detect 11 new stellar stream candidates. In general, these newly detected streams are wider and lower surface brightness than those detected in previous surveys. We find several tentative associations of these new stream candidates with stellar structures detected in other regions of the sky. In addition, we find evidence for extratidal stellar features around four classical globular clusters. The current analysis makes use of $3 \mathrm{yr}$ of DES data. We expect that additional DES observations, improved data reduction techniques, and improved stream detection algorithms will allow fainter and more distant streams to be detected in the near future. While the DES data currently provide the most sensitive wide-area view of the southern sky, they are merely a precursor for larger sky coverage that can be achieved with DECam and, eventually, the Large Synoptic Survey Telescope (LSST). LSST is expected to find $>100$ stellar streams with sensitivity out to the virial radius of the Milky Way (LSST Science Collaboration 2009). These wide-area photometric surveys will greatly expand our ability to probe the Milky Way stellar halo, providing unprecedented insights into Galactic archeology and near-field cosmology.

We thank the attendees at the "Science at the Calyx" event at the Royal Botanic Garden Sydney, as well as the students of St. John's School in North Ryde, Sydney, for their enthusiastic contributions to the process of naming our proposed stellar streams. We likewise acknowledge Aunty Maureen Sulter of the Gamilaraay nation and Mr. Drew Roberts of the Bundjalung nation for their gracious support of, and feedback on, this naming process.

We warmly thank Colegio Antonio Varas in Vicuña, Chile, for organizing the search for aquatic terms from the native Quechua and Aymara cultures. In particular, we thank high school students Dánae Rojas and Emerson Carvajal, their teacher Yeimy Vargas, and dozens of enthusiastic kindergarteners and first graders who selected the Chilean stream names.

N.S. acknowledges support by NASA ATP grant NNH12ZDA001N and NSF grant AST-1412107 and thanks the LSSTC Data Science Fellowship Program; her time as a Fellow has benefited this work. E.B. acknowledges financial support from the European Research Council (ERC-StG335936).

Funding for the DES Projects has been provided by the U.S. Department of Energy, the U.S. National Science Foundation, the Ministry of Science and Education of Spain, the Science and Technology Facilities Council of the United Kingdom, the Higher Education Funding Council for England, the National Center for Supercomputing Applications at the University of Illinois at Urbana-Champaign, the Kavli Institute of Cosmological Physics at the University of Chicago, the Center for Cosmology and Astro-Particle Physics at the Ohio State University, the Mitchell Institute for Fundamental Physics and Astronomy at Texas A\&M University, Financiadora de Estudos e Projetos, Fundação Carlos Chagas Filho de Amparo à Pesquisa do Estado do Rio de Janeiro, Conselho Nacional de
Desenvolvimento Científico e Tecnológico and the Ministério da Ciência, Tecnologia e Inovação, the Deutsche Forschungsgemeinschaft, and the Collaborating Institutions in the Dark Energy Survey.

The Collaborating Institutions are Argonne National Laboratory, the University of California at Santa Cruz, the University of Cambridge, Centro de Investigaciones Energéticas, Medioambientales y Tecnológicas-Madrid, the University of Chicago, University College London, the DES-Brazil Consortium, the University of Edinburgh, the Eidgenössische Technische Hochschule (ETH) Zürich, Fermi National Accelerator Laboratory, the University of Illinois at Urbana-Champaign, the Institut de Ciències de l'Espai (IEEC/CSIC), the Institut de Física d'Altes Energies, Lawrence Berkeley National Laboratory, the LudwigMaximilians Universität München and the associated Excellence Cluster Universe, the University of Michigan, the National Optical Astronomy Observatory, the University of Nottingham, The Ohio State University, the University of Pennsylvania, the University of Portsmouth, SLAC National Accelerator Laboratory, Stanford University, the University of Sussex, Texas A\&M University, and the OzDES Membership Consortium.

Based in part on observations at Cerro Tololo InterAmerican Observatory, National Optical Astronomy Observatory, which is operated by the Association of Universities for Research in Astronomy (AURA) under a cooperative agreement with the National Science Foundation.

The DES data management system is supported by the National Science Foundation under grant nos. AST-1138766 and AST-1536171. The DES participants from Spanish institutions are partially supported by MINECO under grants AYA2015-71825, ESP2015-88861, FPA2015-68048, SEV2012-0234, SEV-2016-0597, and MDM-2015-0509, some of which include ERDF funds from the European Union. IFAE is partially funded by the CERCA program of the Generalitat de Catalunya. Research leading to these results has received funding from the European Research Council under the European Union's Seventh Framework Program (FP7/20072013), including ERC grant agreements 240672, 291329, and 306478. We acknowledge support from the Australian Research Council Centre of Excellence for All-sky Astrophysics (CAASTRO), through project no. CE110001020.

This manuscript has been authored by Fermi Research Alliance, LLC, under contract no. DE-AC02-07CH11359 with the U.S. Department of Energy, Office of Science, Office of High Energy Physics. The United States Government retains and the publisher, by accepting the article for publication, acknowledges that the United States Government retains a nonexclusive, paid-up, irrevocable, worldwide license to publish or reproduce the published form of this manuscript, or allow others to do so, for United States Government purposes.

Facility: Blanco (DECam).

Software: SExtractor (Bertin \& Arnouts 1996), HEALPix (Górski et al. 2005), ${ }^{63}$ astropy (Astropy Collaboration et al. 2013), matplotlib (Hunter 2007), numpy (Van Der Walt et al. 2011), scipy (Jones et al. 2001), healpy, ${ }^{64}$. fitsio, ${ }^{65}$ ngmix (Sheldon 2014), ${ }^{66}$ emcee (Foreman-Mackey

\footnotetext{
63 http://healpix.sourceforge.net

64 https://github.com/healpy/healpy

65 https://github.com/esheldon/fitsio

66 https://github.com/esheldon/ngmix
} 
Table 5

Input Specifications for Stream Fits

\begin{tabular}{|c|c|c|c|c|c|}
\hline Name & $\begin{array}{c}\text { Width } \\
\text { (On) } \\
\text { (deg) }\end{array}$ & $\begin{array}{c}\text { Width } \\
\text { (Off) } \\
\text { (deg) }\end{array}$ & $\begin{array}{l}\text { Separation } \\
\text { (deg) }\end{array}$ & $m-M$ & Age \\
\hline Tucana III & 0.8 & 0.8 & \pm 0.8 & fixed & fixed \\
\hline ATLAS & 1.2 & 1.2 & \pm 1.2 & free & free \\
\hline Molonglo & 1.5 & 3.0 & -2.6 & free & fixed \\
\hline Phoenix & 0.7 & 0.7 & \pm 0.8 & free & fixed \\
\hline Indus & 3.3 & 3.3 & \pm 4.1 & free & fixed \\
\hline Jhelum & 2.5 & 1.2 & +2.0 & free & fixed \\
\hline Ravi & 2.9 & 2.9 & \pm 3.6 & free & free \\
\hline Chenab & 4.0 & 4.0 & \pm 4.7 & free & fixed \\
\hline Elqui & 2.0 & 2.0 & \pm 2.2 & free & free \\
\hline Aliqa Uma & 0.6 & 1.2 & -1.5 & fixed & fixed \\
\hline Turbio & 1.2 & 2.4 & +2.0 & free & fixed \\
\hline Willka Yaku & 0.4 & 0.8 & +0.8 & free & free \\
\hline Turranburra & 2.4 & 2.4 & \pm 3.0 & free & free \\
\hline Wambelong & 2.0 & 2.0 & \pm 2.2 & free & free \\
\hline Palca & $\cdots$ & & & fixed & fixed \\
\hline
\end{tabular}

Note. Input specifications for stellar stream fits. By default, the widths of the on- and off-stream regions are four times the Gaussian stream width. The separation between on and off regions is measured between the stream axis and the center of the off-stream region.

et al. 2013), ugali (Bechtol et al. 2015), ${ }^{67}$ galpot (Dehnen \& Binney 1998), ${ }^{68}$ galstreams (Mateu 2017). ${ }^{69}$

\section{Appendix Stream Fit Configuration}

Table 5 contains the input specifications for the fits to each stream. The procedure for selecting on- and off-stream regions is described in Section 3.3. These regions were derived in a variety of ways in order to optimize foreground subtraction and to avoid contamination by other resolved stellar populations, including other streams, globular clusters, and dwarf galaxies. For the ATLAS stream, when calculating $N_{*}$, we selected a region along the polynomial fit in Equation (6) to account for the curvature of the stream relative to a great circle. Due to the variation in region definitions, we list here the widths and separations of the selected regions for each stream. Additionally, we list the parameters that were fit for each stream. In many cases the data did not allow for a simultaneous fit of all parameters, so a subset of parameters were set to previously determined values or estimated by eye and held fixed.

\section{ORCID iDs}

N. Shipp (10 https://orcid.org/0000-0003-2497-091X

A. Drlica-Wagner (iD https://orcid.org/0000-0001-8251-933X

K. Kuehn (i) https://orcid.org/0000-0003-0120-0808

L. Strigari (i) https://orcid.org/0000-0001-5672-6079

A. K. Vivas (ib https://orcid.org/0000-0003-4341-6172

B. Yanny (D) https://orcid.org/0000-0002-9541-2678

D. Brooks (i) https://orcid.org/0000-0002-8458-5047

F. J. Castander (iD https://orcid.org/0000-0001-7316-4573

J. García-Bellido (i) https://orcid.org/0000-0002-9370-8360

\footnotetext{
$\overline{67}$ https://github.com/DarkEnergySurvey/ugali

68 https://github.com/PaulMcMillan-Astro/GalPot

69 https://github.com/cmateu/galstreams
}

D. Gruen (iD https://orcid.org/0000-0003-3270-7644

R. A. Gruendl (iD https://orcid.org/0000-0002-4588-6517

P. Martini (i) https://orcid.org/0000-0002-4279-4182

R. Miquel (i) https://orcid.org/0000-0002-6610-4836

A. K. Romer (iD https://orcid.org/0000-0002-9328-879X

G. Tarle (i) https://orcid.org/0000-0003-1704-0781

A. R. Walker (1D https://orcid.org/0000-0002-7123-8943

R. H. Wechsler (iD https://orcid.org/0000-0003-2229-011X

\section{References}

Agnello, A. 2017, MNRAS, 471, 2013

Aihara, H., Arimoto, N., Armstrong, R., et al. 2018, PASJ, 70, S4

An, D., Beers, T. C., Johnson, J. A., et al. 2013, ApJ, 763, 65

An, D., Beers, T. C., Santucci, R. M., et al. 2015, ApJL, 813, L28

Astropy Collaboration, Robitaille, T. P., Tollerud, E. J., et al. 2013, A\&A, 558, A33

Balbinot, E., \& Gieles, M. 2018, MNRAS, 474, 2479

Balbinot, E., Yanny, B., Li, T. S., et al. 2016, ApJ, 820, 58

Bechtol, K., Drlica-Wagner, A., Balbinot, E., et al. 2015, ApJ, 807, 50

Bell, E. F., Zucker, D. B., Belokurov, V., Sharma, S., et al. 2008, ApJ, 680, 295

Belokurov, V., Evans, N. W., Bell, E. F., et al. 2007a, ApJL, 657, L89

Belokurov, V., Evans, N. W., Irwin, M. J., Lynden-Bell, D., et al. 2007b, ApJ, 658,337

Belokurov, V., Zucker, D. B., Evans, N. W., et al. 2006a, ApJL, 642, L137

Belokurov, V., Zucker, D. B., Evans, N. W., et al. 2006b, ApJL, 647, L111

Belokurov, V., Zucker, D. B., Evans, N. W., et al. 2007c, ApJ, 654, 897

Bernard, E. J., Ferguson, A. M. N., Schlafly, E. F., et al. 2014, MNRAS, 443, L84

Bernard, E. J., Ferguson, A. M. N., Schlafly, E. F., et al. 2016, MNRAS, 463, 1759

Bertin, E., \& Arnouts, S. 1996, A\&AS, 117, 393

Bertin, E., Mellier, Y., Radovich, M., et al. 2002, in ASP Conf. Ser. 281, Astronomical Data Analysis Software and Systems XI, ed. D. A. Bohlender, D. Durand, \& T. H. Handley (San Francisco, CA: ASP), 228

Besla, G., Kallivayalil, N., Hernquist, L., et al. 2010, ApJL, 721, L97

Besla, G., Kallivayalil, N., Hernquist, L., et al. 2012, MNRAS, 421, 2109

Blumenthal, G. R., Faber, S. M., Primack, J. R., \& Rees, M. J. 1984, Natur, 311,517

Bonaca, A., Geha, M., \& Kallivayalil, N. 2012, ApJL, 760, L6

Bonaca, A., Geha, M., Küpper, A. H. W., et al. 2014, ApJ, 795, 94

Bovy, J. 2014, ApJ, 795, 95

Bovy, J., Bahmanyar, A., Fritz, T. K., \& Kallivayalil, N. 2016, ApJ, 833, 31

Bovy, J., Erkal, D., \& Sanders, J. L. 2017, MNRAS, 466, 628

Bowden, A., Belokurov, V., \& Evans, N. W. 2015, MNRAS, 449, 1391

Bullock, J. S., \& Johnston, K. V. 2005, ApJ, 635, 931

Burke, D., Rykoff, E. S., Allam, S., et al. 2018, AJ, 155, 41

Carballo-Bello, J. A., Martínez-Delgado, D., Navarrete, C., et al. 2018, MNRAS, 474, 683

Carlberg, R. G. 2009, ApJL, 705, L223

Carlberg, R. G. 2012, ApJ, 748, 20

Carlberg, R. G. 2016, ApJ, 820, 45

Carollo, D., Beers, T. C., Chiba, M., et al. 2010, ApJ, 712, 692

Carollo, D., Beers, T. C., Lee, Y. S., et al. 2007, Natur, 450, 1020

Chabrier, G. 2001, ApJ, 554, 1274

Claydon, I., Gieles, M., \& Zocchi, A. 2017, MNRAS, 466, 3937

Dambis, A. K. 2006, A\&AT, 25, 185

Das, P., \& Binney, J. 2016, MNRAS, 460, 1725

Deason, A. J., Belokurov, V., \& Evans, N. W. 2011, MNRAS, 416, 2903

de Grijs, R., \& Bono, G. 2016, ApJS, 227, 5

Dehnen, W., \& Binney, J. 1998, MNRAS, 294, 429

de Jong, J. T. A., Yanny, B., Rix, H.-W., et al. 2010, ApJ, 714, 663

DES Collaboration 2005, arXiv:astro-ph/0510346

DES Collaboration 2016, MNRAS, 460, 1270

DES Collaboration 2017, arXiv:1708.01530

DES Collaboration 2018, arXiv:1801.03181

Diehl, H. T., Neilsen, E., Gruendl, R., et al. 2016, Proc. SPIE, 9910, 99101D

Diemand, J., Kuhlen, M., Madau, P., et al. 2008, Natur, 454, 735

Dinescu, D. I., Girard, T. M., van Altena, W. F., Mendez, R. A., \& Lopez, C. E. 1997, AJ, 114, 1014

Dinescu, D. I., van Altena, W. F., Girard, T. M., \& López, C. E. 1999, AJ, 117,277

Dotter, A., Chaboyer, B., Jevremović, D., et al. 2008, ApJS, 178, 89

Drlica-Wagner, A., Bechtol, K., Allam, S., et al. 2016, ApJL, 833, L5 
Drlica-Wagner, A., Bechtol, K., Rykoff, E. S., et al. 2015, ApJ, 813, 109 Drlica-Wagner, A., Sevilla-Noarbe, I., Rykoff, E. S., et al. 2018, ApJS, 235, 33 Erkal, D., \& Belokurov, V. 2015a, MNRAS, 450, 1136

Erkal, D., \& Belokurov, V. 2015b, MNRAS, 454, 3542

Erkal, D., Belokurov, V., Bovy, J., \& Sanders, J. L. 2016a, MNRAS, 463, 102

Erkal, D., Koposov, S. E., \& Belokurov, V. 2017, MNRAS, 470, 60

Erkal, D., Sanders, J. L., \& Belokurov, V. 2016b, MNRAS, 461, 1590

Fitzpatrick, E. L. 1999, PASP, 111, 63

Flaugher, B., Diehl, H. T., Honscheid, K., et al. 2015, AJ, 150, 150

Foreman-Mackey, D., Hogg, D. W., Lang, D., \& Goodman, J. 2013, PASP, 125,306

Gibbons, S. L. J., Belokurov, V., \& Evans, N. W. 2014, MNRAS, 445, 3788

Gillessen, S., Eisenhauer, F., Trippe, S., et al. 2009, ApJ, 692, 1075

Gnedin, O. Y., \& Ostriker, J. P. 1997, ApJ, 474, 223

Górski, K. M., Hivon, E., Banday, A. J., et al. 2005, ApJ, 622, 759

Grillmair, C. J. 2006, ApJL, 645, L37

Grillmair, C. J. 2014, ApJL, 790, L10

Grillmair, C. J. 2017, ApJ, 847, 119

Grillmair, C. J., \& Carlberg, R. G. 2016, ApJL, 820, L27

Grillmair, C. J., Freeman, K. C., Irwin, M., \& Quinn, P. J. 1995, AJ, 109, 2553

Grillmair, C. J., Jarrett, T. H., \& Ha, A. C. 2004, in ASP Conf. Ser. 327,

Satellites and Tidal Streams, ed. F. Prada, D. Martinez Delgado, \&

T. J. Mahoney (San Francisco, CA: ASP), 276

Hamilton, A. J. S., \& Tegmark, M. 2004, MNRAS, 349, 115

Harris, W. E. 1996, AJ, 112, 1487

Hattori, K., Yoshii, Y., Beers, T. C., Carollo, D., \& Lee, Y. S. 2013, ApJL, 763, L17

Hunter, J. D. 2007, CSE, 9, 90

Ibata, R., Irwin, M., Lewis, G. F., \& Stolte, A. 2001, ApJL, 547, L133

Ibata, R. A., Lewis, G. F., Irwin, M. J., \& Quinn, T. 2002, MNRAS, 332, 915

Jarvis, M., Sheldon, E., Zuntz, J., et al. 2016, MNRAS, 460, 2245

Jethwa, P., Torrealba, G., Navarrete, C., et al. 2017, MNRAS, submitted (arXiv:1711.09103)

Johnston, K. V. 1998, ApJ, 495, 297

Johnston, K. V., Law, D. R., \& Majewski, S. R. 2005, ApJ, 619, 800

Johnston, K. V., Spergel, D. N., \& Haydn, C. 2002, ApJ, 570, 656

Jones, E., Oliphant, T., Peterson, P., et al. 2001, SciPy: Open Source Scientific Tools for Python, Zenodo, doi:10.5281/zenodo.376244, http://www. scipy.org/

Jurić, M., Ivezić, Ž., Brooks, A., et al. 2008, ApJ, 673, 864

Kafle, P. R., Sharma, S., Lewis, G. F., \& Bland-Hawthorn, J. 2013, MNRAS, 430, 2973

Kim, D., \& Jerjen, H. 2015, ApJL, 808, L39

Kirby, E. N., Cohen, J. G., Guhathakurta, P., et al. 2013, ApJ, 779, 102

Koposov, S., Belokurov, V., Torrealba, G., \& Evans, W. 2015, IAUGA, 22, 2256759

Koposov, S. E., Belokurov, V., Evans, N. W., et al. 2012, ApJ, 750, 80

Koposov, S. E., Irwin, M., Belokurov, V., et al. 2014, MNRAS, 442, L85

Koposov, S. E., Rix, H.-W., \& Hogg, D. W. 2010, ApJ, 712, 260

Küpper, A. H. W., Balbinot, E., Bonaca, A., et al. 2015, ApJ, 803, 80

Küpper, A. H. W., Lane, R. R., \& Heggie, D. C. 2012, MNRAS, 420, 2700

Kuzma, P. B., Da Costa, G. S., \& Mackey, A. D. 2018, MNRAS, 473, 2881

Law, D. R., Johnston, K. V., \& Majewski, S. R. 2005, ApJ, 619, 807

Law, D. R., \& Majewski, S. R. 2010, ApJ, 714, 229

Leon, S., Meylan, G., \& Combes, F. 2000, A\&A, 359, 907

Li, T. S., Balbinot, E., Mondrik, N., et al. 2016, ApJ, 817, 135

LSST Science Collaboration 2009, arXiv:0912.0201

Luque, E., Pieres, A., Santiago, B., et al. 2017, MNRAS, 468, 97
Luque, E., Queiroz, A., Santiago, B., et al. 2016, MNRAS, 458, 603

Luque, E., Santiago, B., Pieres, A., et al. 2018, MNRAS, 478, 2006

Majewski, S. R., Kunkel, W. E., Law, D. R., et al. 2004, AJ, 128, 245

Majewski, S. R., Skrutskie, M. F., Weinberg, M. D., \& Ostheimer, J. C. 2003, ApJ, 599, 1082

Mateu, C. 2017, Galstreams: Milky Way Streams Footprint Library and Toolkit, 2018-04-24, Astrophysics Source Code Library, ascl:1711.010

Mateu, C., Read, J. I., \& Kawata, D. 2018, MNRAS, 474, 4112

McMillan, P. J. 2017, MNRAS, 465, 76

Morganson, E., Gruendl, R. A., Menanteau, F., et al. 2018, PASP, 130, 074501

Newberg, H. J., \& Carlin, J. L. (ed.) 2016, Tidal Streams in the Local Group and Beyond, Vol. 420 (Cham: Springer)

Newberg, H. J., Yanny, B., Rockosi, C., et al. 2002, ApJ, 569, 245

Ngan, W. H. W., \& Carlberg, R. G. 2014, ApJ, 788, 181

Nidever, D. L., Majewski, S. R., \& Butler Burton, W. 2008, ApJ, 679, 432

Nidever, D. L., Olsen, K., Walker, A. R., et al. 2017, AJ, 154, 199

Odenkirchen, M., Grebel, E. K., Rockosi, C. M., et al. 2001, ApJL, 548, L165

Olszewski, E. W., Saha, A., Knezek, P., et al. 2009, AJ, 138, 1570

Pawlowski, M. S., McGaugh, S. S., \& Jerjen, H. 2015, MNRAS, 453, 1047

Pawlowski, M. S., Pflamm-Altenburg, J., \& Kroupa, P. 2012, MNRAS, 423, 1109

Pearson, S., Küpper, A. H. W., Johnston, K. V., \& Price-Whelan, A. M. 2015, ApJ, 799, 28

Peebles, P. J. E. 1965, ApJ, 142, 1317

Piatti, A. E. 2018, MNRAS, 473, 492

Pieres, A., Santiago, B., Balbinot, E., et al. 2016, MNRAS, 461, 519

Pieres, A., Santiago, B. X., Drlica-Wagner, A., et al. 2017, MNRAS, 468, 1349

Press, W. H., \& Schechter, P. 1974, ApJ, 187, 425

Price-Whelan, A. M., Hogg, D. W., Johnston, K. V., \& Hendel, D. 2014, ApJ, 794, 4

Rocha-Pinto, H. J., Majewski, S. R., Skrutskie, M. F., Crane, J. D., \& Patterson, R. J. 2004, ApJ, 615, 732

Rockosi, C. M., Odenkirchen, M., Grebel, E. K., et al. 2002, AJ, 124, 349

Rozo, E., Rykoff, E. S., Abate, A., et al. 2016, MNRAS, 461, 1431

Sanders, J. L. 2014, MNRAS, 443, 423

Sanders, J. L., Bovy, J., \& Erkal, D. 2016, MNRAS, 457, 3817

Sanderson, R. E., Vera-Ciro, C., Helmi, A., \& Heit, J. 2016, arXiv:1608.05624

Sandford, E., Küpper, A. H. W., Johnston, K. V., \& Diemand, J. 2017, MNRAS, 470, 522

Schlafly, E. F., \& Finkbeiner, D. P. 2011, ApJ, 737, 103

Schlegel, D. J., Finkbeiner, D. P., \& Davis, M. 1998, ApJ, 500, 525

Schönrich, R., Binney, J., \& Dehnen, W. 2010, MNRAS, 403, 1829

Sheldon, E. S. 2014, MNRAS, 444, L25

Simon, J. D., Li, T. S., Drlica-Wagner, A., et al. 2017, ApJ, 838, 11

Springel, V., Wang, J., Vogelsberger, M., et al. 2008, MNRAS, 391, 1685

Swanson, M. E. C., Tegmark, M., Hamilton, A. J. S., \& Hill, J. C. 2008, MNRAS, 387, 1391

Van Der Walt, S., Colbert, S. C., \& Varoquaux, G. 2011, CSE, 13, 22

Walker, A. R., Kunder, A. M., Andreuzzi, G., et al. 2011, MNRAS, 415, 643

Willman, B., Blanton, M. R., West, A. A., et al. 2005a, AJ, 129, 2692

Willman, B., Dalcanton, J. J., Martinez-Delgado, D., et al. 2005b, ApJL, 626, L85

Yanny, B., Newberg, H. J., Grebel, E. K., et al. 2003, ApJ, 588, 824

Yoon, J. H., Johnston, K. V., \& Hogg, D. W. 2011, ApJ, 731, 58

York, D. G., Adelman, J., Anderson, J. E., Jr., et al. 2000, AJ, 120, 1579

Zucker, D. B., Belokurov, V., Evans, N. W., et al. 2006a, ApJL, 643, L103

Zucker, D. B., Belokurov, V., Evans, N. W., et al. 2006b, ApJL, 650, L41 